\title{
A modified protocol for in vitro maturation of mouse oocytes from secondary preantral follicles
}

\author{
Weidong Wang*, Yaxu Tang, Lujuan Ni, Theerayut Jongwutiwes, Hung-Ching Liu, Zev Rosenwaks \\ The Ronald O. Perelman and Claudia Cohen Center for Reproductive Medicine, Weill Medical College of Cornell University, New \\ York, USA \\ Email: ${ }^{\text {wew2004@med.cornell.edu }}$
}

Received 7 October 2011; revised 12 November 2011; accepted 16 December 2011

\begin{abstract}
A 2-step culture system was designed and tested for the in vitro maturation efficiency of oocytes from pre-puberty preantral follicles of $\mathrm{FVB} / \mathrm{N}$ inbred mice. The following modifications were made: 1) The concentration of ITS was reduced by half in the basal MIF medium to minimize uncoordinated growth between oocyte and GC cells; 2) Heterogeneous preantral follicles were cultured in groups of 3 - 5 follicles in hanging drops of medium with reduced concentration of ITS for six days to induction follicular aggregation. This hanging drop method mimics a 3-D IVM culture system at the early stage of cultivation in which the sphere structure of each follicle is well maintained. It also enables follicles in each aggregate to communicate with each other, synchronize their growth, and thus prevent immature follicular rupture; 3) Medium was further supplemented with retinoic acid to enhance developmental capacity of meiotically arrested oocytes. After a 14-day culture in vitro, 37\% of the collected inbred preantral follicles completed nuclear maturation. Approximately 94\% of the mature oocytes tested were able to be fertilized; and $77 \%$ of them developed into healthy embryos. These results demonstrate that our IVM system is reliable to produce a satisfactory number of high quality oocytes. In addition, multiple cytoplasmic parameters, including gene expression of key regulators, chromosome/spindle organization, mitochondrial proliferation and distribution, and total ATP content were explored to characterize the supportive and limiting components of our IVM system so that the culture system can be further optimized.
\end{abstract}

Keywords: Follicle; Oocyte; In Vitro Maturation; In Vitro Fertilization; Hanging Drops; Fertility; Spindle; Mitochondrion and ATP Content

${ }^{*}$ Corresponding author.

\section{INTRODUCTION}

In vitro maturation (IVM) of human oocytes has gained increasing attention and interest in infertility treatment with great promise [1]. This technique offers an alternative to conventional in vitro Fertilization (IVF) and Embryo Transfer (IVF-ET) by which the high cost of gonadotropin administration can be reduced, particularly for poor responders to gonadotropins; and the side effects associated with gonadotropin stimulation such as ovarian hyperstimulation and possible elevated hormonerelated tumorigenesis can be minimized [2-4]. Different IVM culture systems have been established in several mammalian species to study oocyte development, and have applied to assisted reproduction in humans and livestock animals [5,6]. However, although significant progresses have been achieved in recent years, the current IVM protocols are far from optimal in terms of the number of mature oocytes obtained, as well as fertilization and live birth rates.

Immature mouse oocytes have long been used to develop reliable follicle culture systems and to investigate physiology of folliculogenesis and oocyte maturation. Both enzymatically isolated granulosa-oocyte complexes (GOCs) and mechanically dissected intact follicles had been tested in various 2-D and 3-D culture systems [710]. In general, a 3-D culture system has many advantages over a 2-D system in that the intricate follicular architecture as well as the granulosa-oocyte interaction are well preserved. Maintenance of 3-D structure of IVM follicles has been achieved by various approaches including culturing in membrane inserts, daily transfer of follicles to new droplets, or culturing intact follicles encapsulated with hydrogels like agar/agarose, alginate and hyaluronan [11-13].

A precisely coordinated multi-directional autocrine and paracrine communication between oocytes and somatic cells including granulosa cells, theca cells, as well as certain extra-cellular matrix/gap-junction components, is crucial for oocyte growth and cytoplasmic meiotic 
competence [14-16]. Growth of mammalian oocytes can be divided into three phases according to their developmental stages and responsiveness to gonadotropins: 1) gonadotropin-independent phase in which primordial follicles develop to primary and secondary preantral follicles; 2) gonadotropin-responsive phase in which transition from preantral to early antral stage occurs. At this stage, follicle growth and oocyte maturation are primarily controlled by intrinsic intraovarian signals from oocytes and granulosa cells, although exogenous FSH can stimulate follicular growth; 3) gonadotropin-dependent phase, which includes follicle selection and ovulation [17-20]. Of the three phases, the preantral-early antral transition is most susceptible to follicular atresia [21]. To prevent the oocyte from degeneration at this stage, the cumulus cells continuously provide the oocyte with nutrients and regulatory signals to sustain the progression of maturation, in particular nuclear maturation. Reciprocally, oocyte-secreted factors regulate the growth and differentiation of granulosa cells; subsequently initiate mucification of the cumulus vestment and ovulation [22, 23]. It has long been observed that, during in vitro maturation, although nuclear maturation is complete, cytoplasmic maturation is not assured [24]. It also has been demonstrated that in vivo produced ova are generally of superior quality in terms of production of healthy embryos and offspring [25,26]. Therefore, establishment of an in vitro culture system that can closely recapitulate the in vivo follicular environment has been the focus of much research.

The most important step in IVM of mouse preantral follicles is to remove dictyate arrest of the oocytes, initiate and complete MI to MII transition. In general, oocytes from mice younger than day 15 after birth are meiotically less competent to complete nuclear maturation in vitro when compared with oocytes collected from mice older than 21 days. Consequently, pre-puberty follicles $(<15$ days pp) showed a dramatic decrease in maturation rate with respect to meiotic completion and developmental competence in comparison with the post-puberty ones ( $\geq 21$ days pp) [8,27-30]. This is most likely due to an inadequate accumulation of a large store of mRNA and proteins required to gain competence for meiosis resumption as well as early embryonic development in early preantral follicles [31]. This hypothesis has confirmed by the induction of GVBD in oocytes from young mice by over-expression of both CDK1 and B1 [32,33]. In addition, an established hypothalamus-pituitary-ovary axis may also contribute to the discrepancy in the developmental competence between pre and post-puberty follicles. Possibly, preantral follicles from older mice have already been better primed by signals from pituitary.

It is well known that genetic background influences oocyte developmental competence in the mouse $[34,35]$.
Various genetic strains differ greatly with respect to efficiency of in vitro fertilization, cleavage rate, responsiveness to hormonal milieu, metabolic status, epigenetic changes, $\mathrm{pH}$ regulation, parthenogenetic activation and pre-implantation development [36-40]. The potential interplay of genetic and environmental factors determines the rate of meiotic maturation in vivo or in vitro amongst different mouse strains [41,42]. Currently, almost all of the IVM culturing systems were tested using follicles from hybrid F1 mice. This study aims to present a reliable IVM culturing method for the maturation of early preantral follicles from $\mathrm{FVB} / \mathrm{N}$ inbred mice.

Enlightened by the methodology in embryoid body formation from ES cells, we modified the conventional IVM protocol by culturing premature follicles in hanging drops of medium during early period of cultivation to ease spatial restriction, prevent premature ovulation and synchronize follicular development. At later stage of IVM, the medium was optimized to facilitate meiotic maturation. Maturation rate of pre-puberty preantral follicles from inbred FVB/N mice was examined. Furthermore, multiple parameters showing the dynamics of nuclear/cytoplasmic maturation by IVM were compared with those by in vivo maturation.

\section{MATERIALS AND METHODS}

\subsection{Follicle Harvest}

Preantral follicles were collected from 14-day old FVB/ $\mathrm{N}$ females carrying an Umodl1-BAC-LacZ transgene, named pW224. Umodl1 is a gonadotropin-responsive gene that is specifically expressed in oocytes, early embryos as well as some adult organs (data not shown). The female transgenic mice show no difference in fertility compared with the wild type FVB/N females when number of ovulated oocytes and litter size of live pups were examined. Furthermore, the transgene pW224 can direct the reporter $\beta$-galactosidase to fully recapitulate the endogenous Umodl1 (paper in preparation). Ovaries were removed and transferred to M2 medium (Millipore, USA; MR-015-D). Preantral follicles were isolated by mechanical micro-dissection. Follicles of $90-130 \mu \mathrm{m}$ in diameter with a healthy morphology were selected. Histological analysis showed that almost all of the selected follicles were 2-layered secondary follicles (Figures 2(B)-a and $\mathbf{e}$ ).

\subsection{In Vitro Maturation of Preantral Follicles and Ovulation Induction}

Follicular aggregates were prepared by pooling 3 - 5 dissected follicles into each $25 \mu \mathrm{l}$ droplet of culture medium and incubated in a humidified incubator gassed with $5 \%$ $\mathrm{CO}_{2}$ at $37^{\circ} \mathrm{C}$ for 6 days by the hanging drop method 
(Figure 1). At day 7, the aggregates with a healthy appearance and no sign of premature follicular rupture were transferred to $30 \mu \mathrm{l}$ droplets of culture medium covered with paraffin oil and cultured by the conventional method. Half of the medium in each droplet was replaced with fresh medium every other day. The following media were used in this study: Group A, MIF ( $\alpha$-MEM/GlutaMax-1(Invitrogen/Gibco, USA; 32571 036) + 10\% FBS (Gemini, USA; 100 - 125) + 1/2 ITS (Sigma, USA; I2521) + 100IU/L FSH (Serono, USA; Gona- $\left.\mathrm{f}^{\mathbb{R}} \mathrm{REF} 75 \mathrm{IU}\right)$ for 10 days. At day 11 , additional $0.25 \mathrm{mM}$ ascorbic acid (Sigma, USA; A4034) and $2 \mu \mathrm{m}$ retinoic acid (Sigma, USA; R2625) were added to the MIF basal medium. To initiate ovulation, the MIF was further supplemented with $10 \mathrm{ng} / \mathrm{ml} \mathrm{EGF}$ and $1.5 \mathrm{IU} / \mathrm{ml}$ hCG from day 13 of culture. Group B, MediCult IVM system (ORIGIO, USA; 8221) + 100 IU/L FSH for 12 days. Human chorionic gonadotropin (hCG; Sigma, USA; CS5-1VL) and EGF (Invitrogen, USA; PMG8044) were added to a final concentration of $1.5 \mathrm{IU} / \mathrm{ml}$ and $10 \mathrm{ng} / \mathrm{ml}$, respectively at day 13; Group C, SAGE IVM system (CooperSurgical/SAGE, USA; SPN-0063) + 100 IU/L $\mathrm{FSH}$ for 12 days, followed by $10 \mathrm{ng} / \mathrm{ml} \mathrm{EGF}$ and 1.5 IU/ml hCG stimulation at day 13. Collected GVBD oocytes were further incubated in M16 medium (Millipore, USA; MR-016-D) supplemented with $2 \mu \mathrm{M}$ retinoic acid (RA) for two days to complete meiotic maturation. Follicles/oocytes from Group A were collected at different maturation stages for other assays. Growth of follicle aggregates were monitored and photographed with a Nikon Eclipse Ti-U inverted microscope (Nikon, Japan) controlled by NIS-Elements F3.0 software.

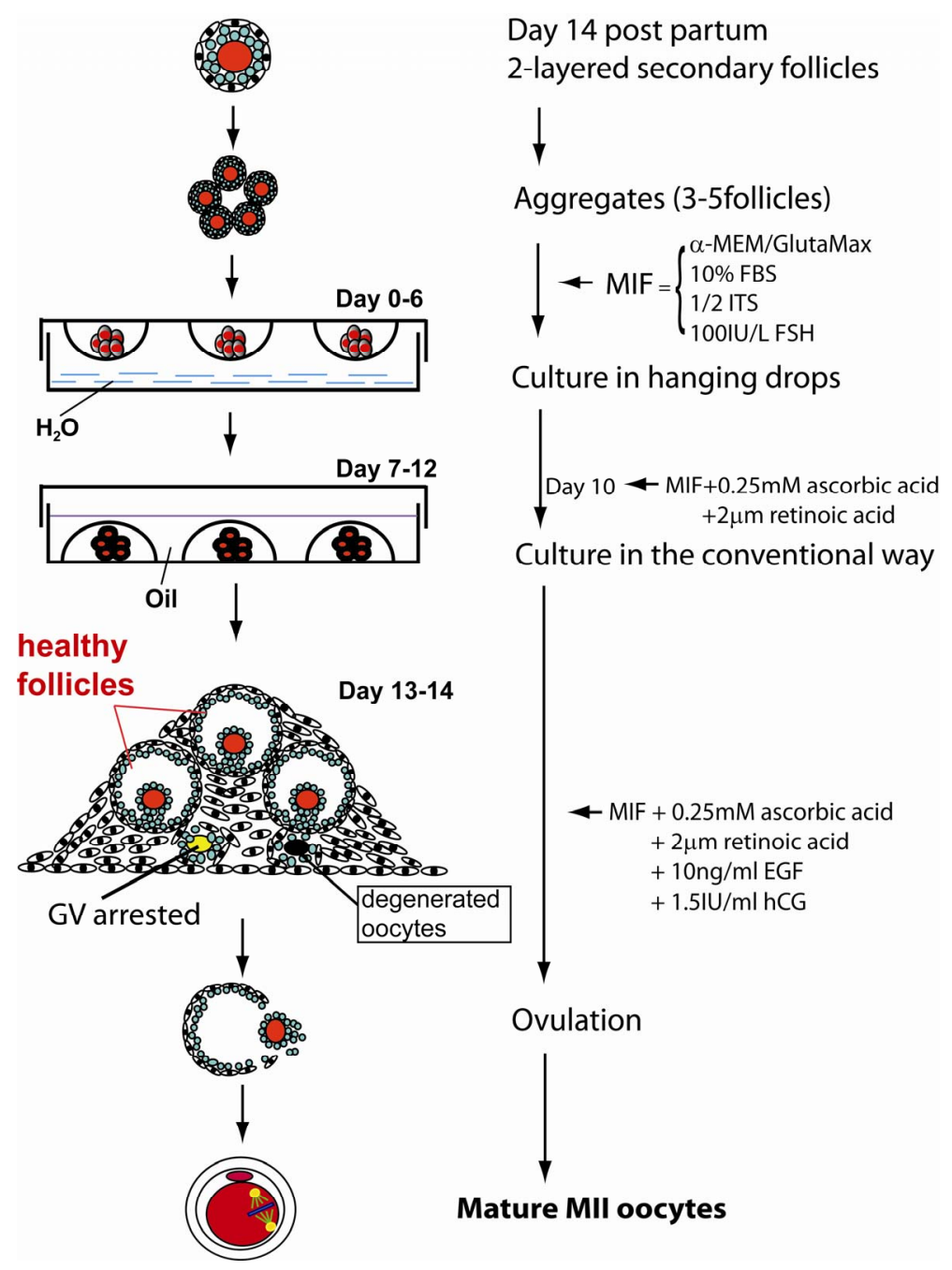

Figure 1. Schematic illustrating experimental design. Two-layered follicles from day 14 female mice were cultured by hanging drop method for six days to facilitate follicular aggregate formation, followed by cultivation in the convention way for six more days. At day 13, MII maturation and ovulation were induced with medium supplemented with retinoic acid, EGF and hCG. 


\section{3. lacZ Staining and Histology}

$\beta$-galactosidase (lacZ) staining on dissected follicles and embryos were performed as previously described $[43,44]$. $\beta$-galactosidase-stained embryos or tissues were dehydrated in graded alcohols, xylene and finally embedded in paraffin and sectioned. After being de-waxed and rehydrated, the sections were counterstained with nuclear fast red (Sigma, USA; N3020) and cover-slipped.

\subsection{RT-PCR}

2-layered $(\mathrm{n}=10)$ and multi-layered $(\mathrm{n}=10)$ preantral follicles were isolated from day 14 in vivo oocytes and IVM aggregates at day 3 , respectively. To isolate oocytes and granulosa/theca cells at the antral stage, IVM antral follicles at day 12 were mechanically ruptured. The released cumulus-oocytes complexes were treated with Hyaluronidase. Ten denuded oocytes were harvested. The remaining granulosa cells and theca cells were pooled. Meanwhile, equal numbers of stage-matched preantral or antral follicles were collected from day 14 and 28 ovaries, respectively, as reference controls for examining the dynamic expression of various ovarian genes during in vivo folliculogenesis. RNAs from mouse R1 embryonic stem (ES) cells and mouse embryonic fibroblast (MEF) cells were also included as controls.

Total RNA from cells, oocytes and follicles was prepared using TRIzol (Invitrogen, USA; 15596-018). Trace amounts of DNA in the RNA samples were removed using DNase I which was then heat inactivated. Reverse transcription was carried out according to the manufacturer's instruction (Promega, USA; A3500). RNA expression levels were quantified using semi-quantitative RT-PCR. cDNA levels were normalized using $\beta$-acitn as an internal control, by which the $\beta$-actin level of each RNA specimen was first checked for equal cDNA synthesis and equal PCR amplification among samples. Primer pair of each gene had been pre-tested to determine the cycle numbers of linear amplification. Primer sequences are available upon request.

The optimal PCR parameters for all genes tested are: 2 min at $92^{\circ} \mathrm{C}$ followed by 28 cycles of $15 \mathrm{sec}$ at $92^{\circ} \mathrm{C}, 30$ sec at $50^{\circ} \mathrm{C}$ and $1 \mathrm{~min}$ at $72^{\circ} \mathrm{C}$. Relative expression levels of the ovarian genes were visualized by agarose gel electrophoresis.

\subsection{TUNEL Assay}

The control $(\mathrm{n}=3)$ and IVM $(\mathrm{n}=3)$ antral follicles were micro-dissected from a day 28 moue ovary and a day 12 follicular aggregate under IVM, respectively. Tissues were first fixed in $4 \%$ paraformaldehyde, followed by dehydration, paraffin embedding, and sectioning. After de-paraffinization and rehydration, selected sections were pre-treated with $10 \mu \mathrm{g} / \mathrm{ml}$ Proteinase $\mathrm{K}$ in $10 \mathrm{mM}$ Tris$\mathrm{HCl}$ for $20 \mathrm{~min}$ at room temperature. Labeling of DNA strand breaks was performed using in Situ Cell Death Detection Kit, Fluorescein (Roche, USA; 11684795910) and analyzed with a Nikon Eclipse 90i fluorescence microscope with appropriate filter and imaging systems.

\subsection{Immunofluorescence Staining}

MI and MII oocytes were briefly exposed to acid Tyrode's (Millipore, USA; MR-004-D) to remove the zona pellucida and fixed in $4 \%$ paraformaldehyde/PBS for $30 \mathrm{~min}$ at room temperature. The oocytes were then permeabilized and blocked in $1 \times$ PBS containing $0.25 \%$ Triton X-100 and $10 \%$ normal goat serum (called blocking solution) for $1 \mathrm{hr}$ at room temperature, followed by an overnight incubation with 1:400 diluted monoclonal anti- $\alpha$-tubulin antibody (Sigma, USA; T9026) in blocking solution at $4^{\circ} \mathrm{C}$. The next morning, samples were washed three times with $0.05 \%$ Tween-20 in $1 \times$ PBS and then incubated with 1:400 diluted AlexaFluor ${ }^{\circledR} 488$ rabbit anti-mouse IgG $(\mathrm{H}+\mathrm{L})$ secondary antibody (Invitrogen, USA; A11059) for $1 \mathrm{hr}$ at room temperature. After three washes in $1 \times \mathrm{PBS} / 0.05 \%$ Tween-20, oocytes were transferred to droplets of mount medium containing DAPI (Santa Cruz, USA; sc24941), cover-slipped and photographed.

To test correct differentiation of inner cell mass and trophoblast cells in the blastocysts derived from IVM oocytes, the blastocysts were double labeled with $\mathrm{Cdx} 2$ and Nanog antibodies. The blastocysts $(\mathrm{n}=3)$ were fixed in paraformaldehyde, permeabilized with $0.25 \%$ Triton X-100/PBS, blocked and incubated with 1:200 diluted primary antbodies against Nanog (Abcam, USA; ab80892) and Cdx2 (BioGenex, USA; Mu392A-UC) overnight at $4 \mathrm{oC}$ followed by two times of wash and a $2 \mathrm{hr}$ incubation with 1:400 diluted secondary antibodies. The secondary antibodies are AlexaFluor ${ }^{\circledR} 488$ goat anti-rabbit $\operatorname{IgG}(\mathrm{H}+$ L) (Invitrogen, USA; A11008) and Texas $\operatorname{Red}^{\mathbb{B}}$-X goat anti-mouse IgG $(\mathrm{H}+\mathrm{L})$ (Molecular Probes, USA; T6390) to detect Nanog and $\mathrm{Cdx} 2$ signals respectively. After three washes with PBS $/ 0.05 \%$ Tween-20, the blastocysts were transferred to the DAPI-containing mounting medium and cover-slipped. Distribution of Nanog and Cdx2positive cells was visualized by fluorescence microscopy.

\subsection{Mitochondrial Staining}

Control and IVM oocytes of MI and MII stages were collected and cultured in M16 medium containing $100 \mathrm{~nm}$ MitoTrack@Red CMXRos (Invitrogen, USA; M7512) for $30 \mathrm{~min}$ at $37^{\circ} \mathrm{C}, 5 \% \mathrm{CO}_{2}$. Oocytes were then rinsed with $1 \times$ PBS once and treated with acid Tyrode's to minimize the signal interference from stained zona pellucida. Oo- 
cytes were further incubated in $\mathrm{M} 16$ at $37^{\circ} \mathrm{C}$ for $1 \mathrm{hr}$ to remove nonspecific signals. Before microscope examination, nuclei were stained with DAPI. Detection of mitochondrial distribution was performed using a Nikon Eclipse 90i fluorescence microscope equipped with a filter set for RITC.

\subsection{ATP Content Measurement}

ATP content in single oocytes was performed using ENLITEN ATP Assay System (Promega, USA; FF2000). A 1:10 serial dilution of the provided ATP standard $\left(10^{-7}\right.$ M) was made. ATP standards at the concentration from $10^{-10}$ to $10^{-16} \mathrm{M}$ were measured to construct the ATP standard curve. IVM oocytes at 2-layered preantral, GV, GVBD and MII stages were collected. Twelve oocytes of each stage were pooled and boiled in $60 \mu \mathrm{l}$ ATP-free $\mathrm{H}_{2} \mathrm{O}$ to release ATP. In addition to the IVM oocytes, stage-matched oocytes from 28-day old untreated females and 21-day old gonadotropin (PMSG, 5IU/female) stimulated females were also included in this assay as well as the Mitochondrial DNA copy number assay to be mentioned later.

The ATP standards and oocyte samples were taken up in $20 \mu \mathrm{l}$ and mixed with equal volume of reconstituted $\mathrm{rL} / \mathrm{L}$ reagent from the Kit. RLU signal was measured with a Luminometer GloMax20/20 (Promega, USA). Each sample was analyzed in three replicates and ATP quantity in each oocyte was deduced from the ATP standard curve. Data are presented in Mean \pm SD.

\subsection{Absolute Mitochondrion Number by Quantitative PCR (Q-PCR)}

Mitochondrial DNA (mtDNA) extraction. Pooled oocytes ( $\mathrm{n}=9$ for each stage) of the same maturation stage were transferred to $200 \mu \mathrm{l}$ thin wall PCR tubes and lysed for $12 \mathrm{hr}$ in mtDNA extraction buffer $(50 \mathrm{mM}$ Tris- $\mathrm{HCl}$, pH 8.5 with $0.5 \%$ Tween-20) containing $100 \mu \mathrm{g} / \mathrm{ml}$ Proteinase $\mathrm{K}$ at $55^{\circ} \mathrm{C}$, followed by heat inactivation at $95^{\circ} \mathrm{C}$ for $10 \mathrm{~min}$ in a total volume of $30 \mu \mathrm{l}$ of reaction.

Standard curve construction. Absolute quantification of oocyte mitochondria was performed by the standard curve method. A 736 bp mtDNA fragment was PCR amplified from mitochondrial genome between nt3455 and nt4191 using oligo 644 (5'CGCCCTAACAACTATTATCTTCCT3') and Oligo 648 (5'GACCGTTTGTTTGTTGTTGAAA3'). This template was purified by gel extraction (Qiagen, USA; QIAEXII,) and quantified by UV spectrophotometry. $1 \mathrm{ng}$ of $736 \mathrm{bp}$ PCR product comprised $1.24 \times 10^{9}$ molecules of double stranded DNA. Real-time PCR amplifications over a copy number range of $1.0 \times 10^{2}$ to $1.0 \times 10^{7}$, which range falls to the $10^{7}$ $10^{2}$ fold dilutions of $1 \mathrm{ng} / \mu 1$ template respectively, were performed. The relationship between the $\mathrm{Ct}$ value and the logarithm of the initial copy number of the standards should be linear with the correlation coefficient $r^{2}>0.99$.

Setup of Q-PCR. All oocyte samples and standards were measured in triplicates in a 364-well PCR plate. Nested primer pair, Oligo 645 (5'GAGCATCTTATCCACGCTTCC3') and Oligo 646 (5'GGTGGTACTCCCGCTGTAAA3') spanning nt3571 to nt3698 of the mitochondrial genome were used to amplify both the standards and oocyte samples at the final concentration of $400 \mathrm{~nm}$. Real-time PCR amplifications were performed in $25 \mu$ l reaction volumes using SYBR green (Applied Biosystems, USA; 4309155) on an ABI-7900 HT cycler (Applied Biosystems, USA). The PCR parameters are: $95^{\circ} \mathrm{C}$ for $2 \mathrm{~min}, 35$ cycles of $95^{\circ} \mathrm{C}$ for $10 \mathrm{~s}$, $55^{\circ} \mathrm{C}$ for $15 \mathrm{~s}, 72^{\circ} \mathrm{C}$ for $20 \mathrm{~s}$, followed by $3 \mathrm{~min}$ at $72^{\circ} \mathrm{C}$.

Data analysis. Threshold cycle number $(\mathrm{Ct})$ was calculated with ABI 7900HT SDS 2.3 software. The quantities of each PCR product were calibrated by a linear regression model using standard curves calculated between $\mathrm{Ct}$ value and the logarithm of the concentration of standards. Student's $t$-test and one-way analysis of variance were performed on mtDNA copy number after logarithmic transformation of the data. The difference in mtDNA copy number between IVM oocytes and either of the two control groups was analyzed by Student's $t$-test. $P$ values less than 0.05 were considered to be significantly different. mtDNA copy number is presented as mean $\pm \mathrm{SD}$.

\subsection{In Vitro Fertilization (IVF) and Embryo Oviduct Transfer}

Collection of fresh sperm. A male C57BL/6J was sacrificed. Epididymides along with the vas deferentia were dissected out and placed in a dish filled with HTF medium (Millipore, USA; MR-070-D). The epididymis was minced. The sperm dish was incubated at $37^{\circ} \mathrm{C}, 5 \% \mathrm{CO}_{2}$ for $1 \mathrm{hr}$. Sperm count was adjusted to $2.5 \times 10^{6} \mathrm{sperm} / \mathrm{ml}$ in $\mathrm{HTF}$.

Piezo-driven zona-drilling on IVM oocytes. Piezodriven zona-drilling was essentially performed with a Prime Tech PMM controller as previously described [45] with some modification in which blunt-tip pipettes in 10 $20 \mu \mathrm{m}$ diameter were used as the piezo-activated drilling pipettes. One or two holes were induced to each oocyte. Prior to IVF, oocytes were recovered in KSOM + AA (Millipore, USA; MR-121-D) for $1 \mathrm{hr}$ in a $37^{\circ} \mathrm{C}$ incubator gassed with $5 \% \mathrm{CO}_{2}$.

IVF. Thirty zona-drilled oocytes were pooled in one $500 \mu \mathrm{l}$ drop of HTF and mixed with $10 \mu \mathrm{l}$ of the C57BL/ $6 \mathrm{~J}$ sperm. After 6 hours of incubation at $37^{\circ} \mathrm{C}$ under $5 \%$ $\mathrm{CO}_{2}$, oocytes were washed with $\mathrm{KSOM}+\mathrm{AA}$ three times and incubated overnight. Fertilized eggs were removed at 2-cell, 3-cell, 4-cell, morula and blastocyst stages for lacZ staining or immunofluorescence labeling. 
The remaining embryos were transferred to the oviducts of two pseudo-pregnant CD-1 female mice. Embryos were then collected at E9.5 and E11.5 for lacZ-staining and genomic genotyping.

\subsection{Genomic PCR}

Genotype of the embryos derived from IVM oocytes was verified by genomic PCR around the region of the polymorphic marker D1Mit3. Genomic DNA was extracted from the yolk sacs attached to the embryos by PK digestion and phenol/chloroform purification. Oligo 642 (5'TTTTTGTTTTCTTTTCTTTTCCC3') and Oligo 643(5'CCCTCTTCTG GTTTCCACAT3') are the primer pair to distinguish the 185 bp FVB and 160 bp C57BL/6 J-specific alleles in this analysis. The thermal cycling conditions are $92^{\circ} \mathrm{C}$ for $3 \mathrm{~min}, 35$ cycles of $92^{\circ} \mathrm{C}$ for $15 \mathrm{~s}, 42^{\circ} \mathrm{C}$ for $30 \mathrm{~s}$ and $68^{\circ} \mathrm{C}$ for $1 \mathrm{~min}$.

\section{RESULTS}

In our pilot studies, we have tried various IVM techniques including the 2-D micro-droplet culture on tissue culture dishes and hydrogel encapsulated 3-D systems to find out the best system for culturing preantral follicles in vitro. The most common adverse event, regardless of which system was tested, is the spontaneous release of the oocyte and follicular degeneration, which normally takes place at days 6 - 8 of culture. Mechanisms underlying the premature oocyte release are not clear yet; possible due to uncoupled growth and differentiation of oocyte and granulosa cells. This protocol makes the following modifications: 1) The concentration of ITS was reduced by half in the basal MIF medium to minimize uncoordinated growth between oocytes and GC cells; 2) Heterogeneous preantral follicles were cultured in groups of 3 - 5 follicles in hanging drops of medium for six days to induce follicular aggregation. This hanging drop method mimics a 3-D IVM culture system at the early stage of cultivation in which the sphere structure of each follicle is well maintained. It also enables follicles in each aggregate to communicate with each other, synchronize their growth, and thus prevent immature follicular rupture; 3) Medium was further supplemented with retinoic acid to enhance development capacity of meiotically arrested oocytes [46-49]. Figure 1 summarizes the IVM procedures.

\subsection{In Vitro Development of Follicles and Nuclear Maturation of Oocytes}

Isolated follicles with a diameter of $90-130 \mu \mathrm{m}$ were collected for this study. The mean diameter $( \pm \mathrm{SD}, \mathrm{N})$ of the enclosed oocytes was $60( \pm 8,30) \mu \mathrm{m}$ (Figure 2(A)a). Histological analysis of these follicles after 1 day of culture showed that they were 2-layered preantral follicles (Figures 2(B)-a and e). On day 3 of culture, follicular aggregates with a healthy appearance formed in all individual hanging drops (Figure 2(A)-b). Progressive enlargement of the aggregates indicated the growth of the IVM follicles. On day 6 of culture, the size of oocytes $(\mathrm{n}=55)$ increased to $65 \pm 8 \mu \mathrm{m}$ in diameter. Accordingly, the cultured follicles grew to a range from 140 to $270 \mu \mathrm{m}$ in diameter, suggesting the heterogeneity in developmental potential among the collected preantral follicles (Figure 2(A)-c). Surprisingly, among 223 follicles under IVM, only three showed premature oocyte release before day 6 , strongly suggesting that culture of follicles in hanging drops, together with reduced ITS, can effectively prevent the spontaneous follicular rupture and follicle degeneration so troublesome to many culture systems. Accelerated growth was observed between days 7 to 9 . Also, the follicles became more homogeneous in size, exhibiting a diameter of $389 \pm 40 \mu \mathrm{m}$ (Figure 2(A)d). The mean diameter $( \pm \mathrm{SD})$ of the enclosed oocytes reached to $70( \pm 3) \mu \mathrm{m}$. From day 13 of culture, just one day after hCG/EGF induction, ovulation could be detected (arrows in Figures 2(A)-e and f). On day 14 of culture, mature MII oocytes were collected from the medium droplets (arrowheads in Figures 2(A)-f and g). A representative photo of oocyte nuclear maturation was shown in Figure 2(A)-g, in which approximately $40 \%$ of collected oocytes developed to the MII stage (arrowheads in Figure 2(A)-g); whereas the rest either remained at GVBD or degenerated at day 14 (arrowhead in Figure 2(A)-g). Prolonged incubation of oocytes in the presence of hCG and EGF resulted in two-cell embryos possibly by parthenogenetic activation (Figure 2(A)-h). Progression of nuclear maturation is summarized in Table 1 (experimental group A). During the long-term culture of 14 days, only 9 follicles degenerated as a result of premature oocyte release. Approximately $96 \%$ of the follicles retained their oocytes within the follicles and displayed vigorous follicular expansion throughout the culture period. Of the 223 follicles collected, $18 \mathrm{MII}$ oocytes $(8 \%)$ were obtained on day 12 of culture without hCG treatment. After a 48-hr hCG induction, additional 65 oocytes $(29 \%)$ showed meiosis maturation as manifested by the extrusion of $1^{\text {st }}$ polar body. In summary, the cumulative nuclear mature rate of $\mathrm{FVB} / \mathrm{N}$ inbred preantral follicles under IVM is $37 \%$.

On day 14 of culture, $40 \%$ of the collected oocytes (n $=90)$ proceeded to meiosis I (MI) as marked by germinal vesicle breakdown (GVBD), but failed to proceed to MII (Table 1). To test whether these GVBD oocytes were able to resume meiosis upon proper activation, we selected 37 GVBD oocytes for zona drilling and subsequent insemination by IVF. Surprisingly, meiosis reinitiated as indicated by PB1 extrusion in 21 sperm-pene- 
trated and 10 unpenetrated oocytes. Further development of the oocytes to the two-cell and blastocyst stages was observed in $90 \%$ of the sperm-penetrated oocytes. This suggests that oocytes blocked at MI during maturation had already gained certain degrees of competence for MII completion, and could be reactivated in respond to signals from sperm.

Morphological and histological features of the maturing follicles were further examined in details. Follicles were mechanically dissected on different days of culture, subjected to lacZ-staining and sectioned. Progressive growth of lacZ-stained follicles was shown in Figure 2(B). Antrum-like cavity formation was initially recognized from day 5. At this stage, oocytes of GV stage were enclosed by proliferating granulosa cells (Figures 2(B)-f-h). By day 12, well-developed antral like cavities were seen in $90 \%$ of growing follicles in which oocytes of GVBD were retained in most cases (Figures 2(B)-i and j). On day 14, upon hCG/EGF stimulation, healthy MII oocytes with extruded PB1 were obtained

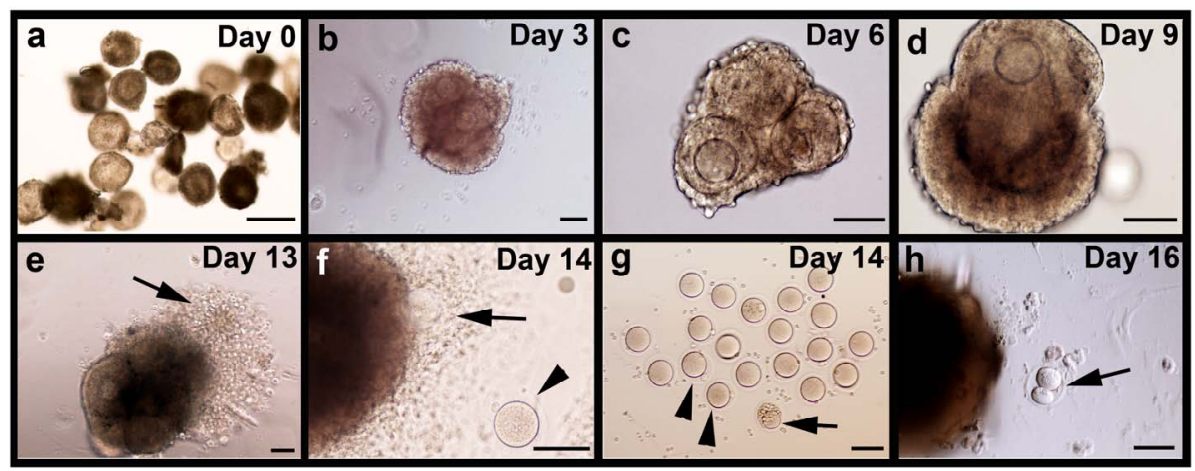

(A)
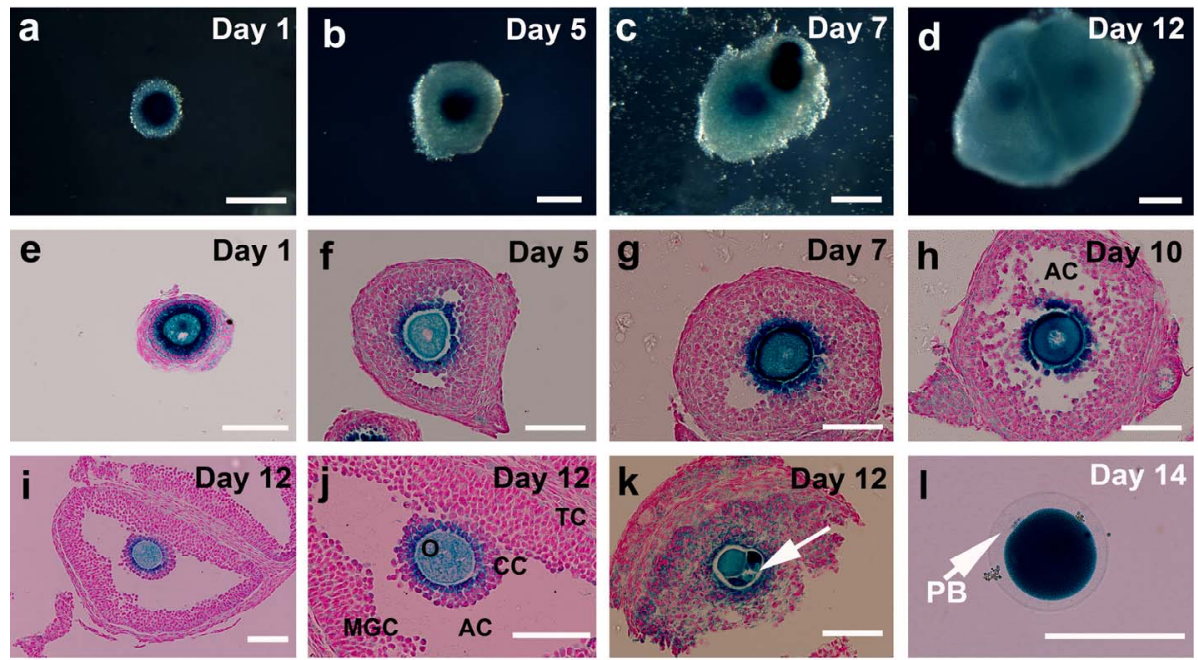

(B)

Figure 2. Progressive development of the follicles and enclosed oocytes in the follicular aggregates. (A) Morphology of the follicular aggregate whole mounts at different culturing stages. (a) 2-layered preantral follicles were collected from 14-day old FVB mice; (b)-(d) In vitro growth of follicles in our MIF culture system; (e)-(f) From day 13, once stimulated by EGF and hCG, maturing follicles started ovulating and matured MII oocytes were released; (g) Shown are some of the oocytes of various stages collected at day 14; (h) Prolonged incubation induced parthenogenesis; (B) Detailed morphological changes of maturing follicles by histological analysis; (a)-(d) Growing follicles from different stages were mechanically segregated and stained by $\beta$-galactosidase (lacZ) activity. Whole mounts of the stained follicles were embedded in paraffin and sectioned; (e) Secondary follicle; (f)-(g) Multi-layered follicles; (h) Follicles undergoing antral formation; (i) A healthy preovulatory follicle at day 12; (j) A higher power view of (i) showing an oocyte surrounded by cumulus cells (CC), an antral cavity(AC), and mural granulose cells (mGC); (k) A fragmented oocyte enclosed in a multi-layered follicle; (1) A lacZ-stained mature MII oocyte. Abbreviations: $\mathrm{AC}$, antral cavity; $\mathrm{CC}$, cumulus cells; MGC, mural granulosa cells; O, oocyte; $\mathrm{PB}$, polar body; TC, theca cells. 
Table 1. Comparison of different media in oocyte maturation from preantral follicles.

\begin{tabular}{|c|c|c|c|c|c|c|c|c|c|c|}
\hline \multirow{2}{*}{ Experimental group } & \multirow{2}{*}{ Medium } & \multirow{2}{*}{$\begin{array}{l}\text { Follicles } \\
\text { collected }\end{array}$} & \multicolumn{4}{|c|}{$\leq 12$-day culture } & \multicolumn{4}{|c|}{$<48 \mathrm{hr}$ after hCG (total) } \\
\hline & & & GV & GVBD & Mature & Degn. & $\mathrm{GV}$ & GVBD & Mature (\%) & Degn. \\
\hline A & MIF & 223 & 67 & 119 & 18 & 19 & 18 & $90^{+}$ & $83\left(37^{*}\right)$ & 32 \\
\hline $\mathrm{B}$ & MediCult & 92 & 28 & 10 & 0 & 54 & 18 & 15 & $0(0)$ & 59 \\
\hline $\mathrm{C}$ & SAGE & 102 & 38 & 38 & 1 & 25 & 27 & 42 & $3(3)$ & 30 \\
\hline
\end{tabular}

*The percentage was calculated based on the total number of follicles collected. ${ }^{+} 37$ were tested for sperm-activation. Degn., degenerated.

(Figure 2(B)-I). It is noteworthy to mention that almost all of the degenerated and GV oocytes were found in follicles that were in direct contact with the surface of the tissue culture dishes (Figure 2(B)-k). Their culture milieu was more like that of a 2-D system, which possibly led to a compromised developmental capacity as compared with those growing on top of them.

In parallel, we repeated the aforementioned experiments by replacing our MIF basal medium with either SAGE or MediCult medium to test the success rate of oocyte in vitro maturation from preantral follicles. Although optimized for the IVM of human oocytes, the two commercially available media failed to produce any significant number of MII oocytes ( $p<0.01$; groups $B$ and $\mathrm{C}$ in Table 1).

\subsection{Cytoplasmic Maturation of Oocytes under IVM}

Terminal deoxynucleotidyl transferase dUTP nick end labeling (TUNEL) assay was carried out to compare the apoptotic activity of the IVM antral follicles with that of stage-matched follicles from in vivo ovaries. Significantly, more TUNEL-positive cells were found in the cumulus-oocyte complex under IVM. Elevated apoptosis in the somatic cells including cumulus cells, mural cumulus cells and theca cells demonstrates that the in vivo maturation conditions are always superior to in vitro culture systems (Figure 3).

Oocytes actually acquire cytoplasmic and nuclear competence within the follicles prior to resumption of meiosis. Substantial growth of oocytes is evident from preantral to GVBD stage while associated granulosa cells continue their growth and differentiation up to the ovulatory stage followed by apoptosis or reprogramming after ovulation. A complex interplay of endocrine, paracrine as well as direct communication between somatic cells and oocytes has to be well orchestrated to assure a well coordinated maturation between cytoplasm and nucleus. To characterize the supportive and limiting components of our IVM system, various parameters of cytoplasmic maturation of the IVM oocytes, including the dynamic expression of key developmental genes, metabolic status and spatial organization of organelles,

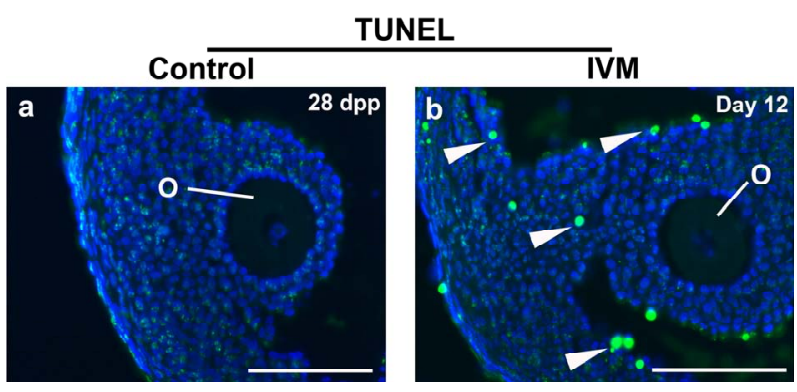

Figure 3. TUNEL assay to detect apoptosis between the control and IVM antral follicles. The control antral follicles were retrieved from a 28 day-old ovary. DNA strand breaks were visualized by detecting flurorescein-dUTP signals. Nuclei were stained with DAPI. Abbreviations: dpp, days postpartum; others as per Figure 2. Scale bars, $100 \mu \mathrm{m}$.

were accessed by comparing with those of in vivo oocytes.

Semi-quantitative PCR. Two members of TGF- $\beta$ superfamily secreted by the oocytes, Growth differentiation factor $9(G d f-9)$ and its homolog bone morphogenetic protein 15 (BMP-15), are the two key proteins in primordial follicle recruitment, granulosa cell proliferation and differentiation, and steroid synthesis [50,51]. Both genes showed a moderate increase from 2-layered preantral follicles to multi-layered follicles and remained steady in oocytes maturated in vivo. Weak signals of BMP-15 were also detectable in granulosa/theca cells. Except a notable down-regulation of $B M P-15$ in the granulosa cells of antral follicles, the IVM system is able to maintain the correct expression patterns of $G d f-9$ and $B M P-15$ as compared with in vivo follicles/oocytes (Figure 4). $M a$ ter, also called NLrp5, encodes a maternal oocyte protein that is required for early embryogenesis. In vivo, expression of Mater barely showed any change once it was turn on from the preantral stage, which was also observed in the IVM oocytes. Expression of ZP3 was significantly up-regulated during the development from 2-layered to multi-layered follicles, and became unchanged thereafter in vivo. Although a similar dynamic pattern was seen in our IVM follicles, the increase of ZP3 was relatively moderate when compared with in vivo follicles. Like $Z P 3$, Uromodulin-like 1(Umodl1) encodes a glycosylated surface protein in oocytes and adjacent cumulus cells (Figure 2). 


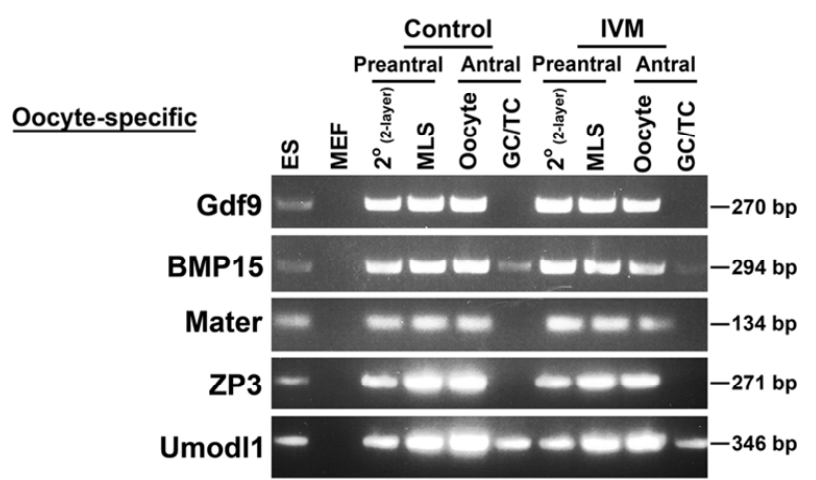

Auto/paracrine

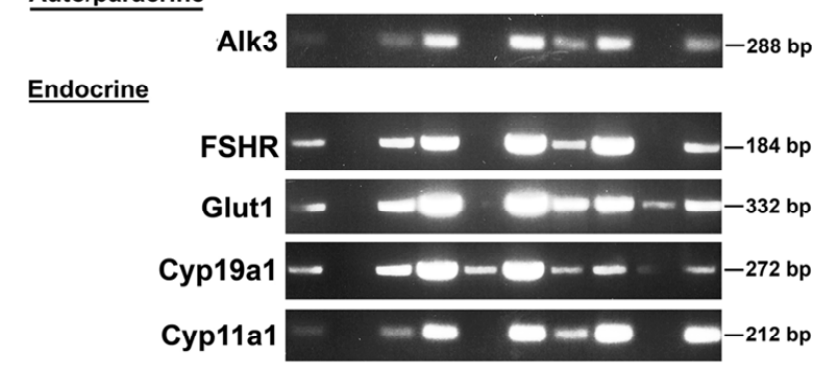

Cell-junction/ECM

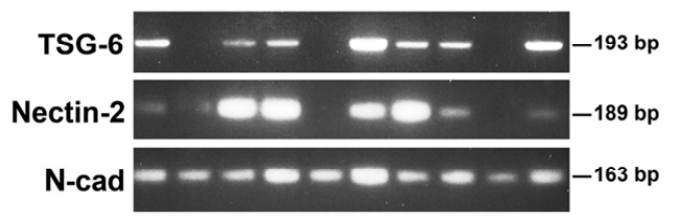

Apoptosis

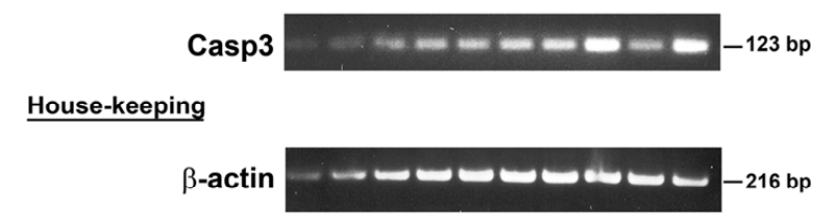

Figure 4. Semi-quantitative RT-PCR to examine the expression profile of ovarian genes in different follicles and cell types that were undergoing in vitro maturation. Stage-matched follicles/oocytes from control (WT) ovaries were included. Ovary-specific genes tested are indicated at the left of the panel and the sizes of amplified PCR fragments are listed at the right. The house-keeping gene, $\beta$-actin was used as the internal control. Abbreviations: ES, embryonic stem cells; MEF, mouse embryonic fibroblast cells; $2^{\circ}$, 2-layered secondary follicles; MLS, multi-layered secondary follicles; GC, granulosa cells; TC, theca cells.

Umodl1, a likely novel component of zona pellucid, may also mediate gonadotropin signaling. Expression of Umodl 1 was up-regulated as oocytes increased in size when in vivo follicles/oocytes were examined; while no dramatic difference in expression patterns was seen between in vivo and in vitro follicles/oocytes. Paracrine signals from oocytes exert their actions via receptors located on the neighboring granulosa cells. Alk3, also known as BMP receptor $1 \mathrm{~A}$, is one of the effector molecules involving in the BMP-15 signaling pathway to regulate the prolifera- tion and differentiation of granulosa cells. Level of Alk3 increased as the granulosa cells proliferate in both the in vivo and in vitro follicles. However, the steady-state expression of Alk3 in the IVM granulosa/theca cells of antral stage was notably lower than the stage-matched control.

Development of the cumulus cell phenotype in vitro is synergistically promoted by signals from oocytes and exogenous FSH. In vivo, concomitant with follicle expansion was an increase of the FSH receptor gene (FSHR) solely in the developing granulose cells. In vitro, FSHR did exhibit a significant increase in expression during the growth of follicles from 2-layered to multi-layered stage. However, its expression in the preovulatory granulosa cells somehow declined as compared with the stagematched control cells. Down-regulation in gene expression was also observed in Glut 1 and Cyp $19 a 1$ in the IVM follicles. Glucose is the main energy substrate in the mammalian ovaries, and its uptake is primarily mediated by a number of glucose transporters (GLUT). Glut 1 is one of the important genes involving in granulosa cell metabolism. Both estradiol and EGF play a role in regulating Glu1 expression. Hypoxia can also increase Glu1 levels and glucose uptake. Interestingly, unlike the in vivo controls whose Glu1 expression increased notably from preantral stage onwards, Glut 1 of the IVM follicles changed minimally over the same time period. Cyp19a1, also known as Aromatase, belongs to the cytochrome P450 superfamily. This enzyme catalyzes the last steps of estrogen synthesis from androgens. Its expression in the IVM follicles was down-regulated in all cell types examined. Another member of the P450 superfamily, Cyp $11 a 1$ was also included in this study. Cyp11a1 protein localizes to the mitochondrial inner membrane and catalyzes the conversion of cholesterol to pregnenolone, the first and rate-limiting step in the synthesis of the steroid hormones. In mammalian ovaries, Cyp1la1 is also a key player in the proliferation and differentiation of theca/mural cumulus cells. No difference in the dynamic expression of Cyp $11 a 1$ could be seen between in vivo and in vitro-cultured follicles/oocytes.

During oogenesis and folliculogenesis, cell-cell and cell-matrix interactions have a profound effect on the availability and actions of hormones and growth factors produced from different cellular compartments including oocytes, granulosa and theca cells. Extracellular matrix (ECM) molecules provide not only structural support to maintain the integrity of developing follicles, but also play an indispensable role in coordinating various signaling pathways. ECM molecules, matrix metalloproteinases and tissue inhibitors of metalloproteinase are usefully markers of follicular health, at least in vitro [52]. Tumor necrosis factor-induced protein-6 (TSG-6) is a member of the hyaluronan-binding protein family. TSG6 
null mice provide us a good example of how ECM integrity affects follicular function. Inactivation of TSG6 gene leads to shedding of cumulus cells, absence of cumulus matrix expansion and eventually "naked" oocytes. As a result, their fertility is severely compromised [53]. In addition to its role in follicle growth, ECM is essential for oocyte ovulation, follicular repair after ovulation, and subsequent corpus luteum formation [54]. In general, the expression patterns of TSG-6 were not significantly different between in vivo and in vitro follicles/oocytes, suggesting our IVM system is able to maintain the integrity of maturing follicles. In contrast, the gene encoding cell-cell adhesion molecule Nectin-2 was down-regulated in the multi-layered preantral follicles and antral granulosa cells under IVM. In vivo expression of Nectin-2 is restricted to the granulosa cells surrounding oocytes. Similarly, N-cad, another gene encoding a cell-cell adhesion molecule, showed a slight decrease in expression under the IVM conditions. Therefore, our IVM system imposed different influences between ECM and cell-cell adhesion molecules in regulating the dynamic morphological changes as well as the growth of follicles (Figure 4).

Apoptosis, or programmed cell death, is a process of self-destruction under physiological conditions. Typical features of cells undergoing apoptosis are cell shrinkage, lipid membrane blebbing, nuclear chromatin condensation and fragmentation, DNA fragmentation and segmentation of cells into apoptotic bodies [55]. Several members of the Bcl-2 and Caspase families have been demonstrated to participate in the induction, execution or suppression of apoptosis. One of the apoptotic genes, Casp 3 was tested in the control and IVM follicles. A moderately higher level of Casp 3 was detected in the granulosa cells of multi-layered preantral and antral follicles under IVM, which was consistent with what we observed by TUNEL assay (Figure 3). A basal level of Casp 3 activity was present in both the control and IVM oocytes (Figure 4).

Immunostaining of spindle. In addition to examining ovarian gene expression and apoptosis levels in the IVM follicles, many other measures to evaluate oocyte quality have been proposed to establish an acceptable criterion for oocyte nuclear and cytoplasmic maturation at the cellular and molecular levels. Extrusion of first polar body (PB1) is considered to be the standard indication for oocyte nuclear maturity, whereas spindle integrity and mitochondrial distribution are commonly used as an index for oocyte cytoplasmic maturity [56-61]. The normal mouse MI spindle has a characteristic barrel shape, is eccentrically placed in the oocyte with the chromosomes being arranged on the metaphase plate at the equator (Figure 5(A)-a). At the MII stage, a typical spindle stretches further and the spindle poles become more focused (Figure 5(A)-c). In general, spindle configuretion was well preserved in the IVM oocytes (Figures 5(A)-b and d). Chromosomes were aligned in a compact metaphase plate at the equator of the spindle. The minor morphological change was that the MI and MII spindles were compressed and stretched into a shape more like a diamond in the IVM oocytes (Figures 5(A)-a-d), suggesting that the IVM conditions affected spindle phenotype.

Mitochondrial distribution and absolute mitochondrial copy number. Coordinated movements of chromosomes and organelles are required for the oocyte maturation. At the time of germinal vesicle break down (GVBD), the chromosomes condense and align themselves on the first meiotic spindle. Meanwhile, a large number of mitochondria move to surround the nucleus and then accompany the spindle to the cortex of the oocyte $[62,63]$. During the transition from the GV to MI stage, mitochondria started aggregating around the condensing chromosome (Figure 5(B)-a). In mature MII oocytes, mitochondria formed a crescent around the condensed MII chromosomes/spindle (Figure 5(B)-c). As expected, the same dynamic patterns of mitochondrial polarization were observed in the IVM oocytes as the controls (Figure 5(B)). However, it is also evident that stronger mitochondrial signals were detected in all of the IVM oocytes examined than those in the stage-matched controls, indicating that our IVM system boosted mitochondrial biosynthesis. Absolute mitochondrial number was quantified by Q-PCR. mtDNA copy number was significantly affected by maturation status and culture conditions. Changes in mtDNA copy number during oocyte maturation are depicted in Figure 5(C). Under the real physiological conditions (in vivo), mtDNA was progressively amplified from $0.38 \pm 0.20 \times 10^{5}$ copies/2-layered preantral oocyte to $0.52 \pm 0.28 \times 10^{5}$ copies/multi-layered GV oocyte, $1.30 \pm 0.51 \times 10^{5} / \mathrm{MI}$ oocyte and $1.89 \pm 0.11 \times$ $10^{5}$ copies/mature MII oocyte. In the IVM group, a drastic ( $\sim 10$ fold) increase in the mtDNA copy number was seen in maturing oocytes from preantral $(0.38 \pm 0.20 \times$ $\left.10^{5}\right)$ to $\mathrm{GV}\left(3.52 \pm 0.10 \times 10^{5}\right)$ stage. On average, a matured IVM oocyte harbored $4.15 \pm 0.36 \times 10^{5}$ copies of mitochondria in its cytoplasm, 1.2-fold higher than an oocyte matured in vivo $(\mathrm{p}<0.001)$. Stage-matched oocytes collected from pregnant mare's serum gonadotropin (PMSG; Sigma, USA; G4877)-primed mice were also included as controls in this study. A relatively moderate increase in mitochondria number among the gonadotropin-stimulated oocytes of various stages suggests that gonadotropin alone can inflate mtDNA synthesis even though it was not as effective as the combinatorial signals provided by our IVM system (b in Figure 5(C)). As predicted, when the ATP content of oocytes from in vivo, IVM and PMSG-simulated groups was investigated, 

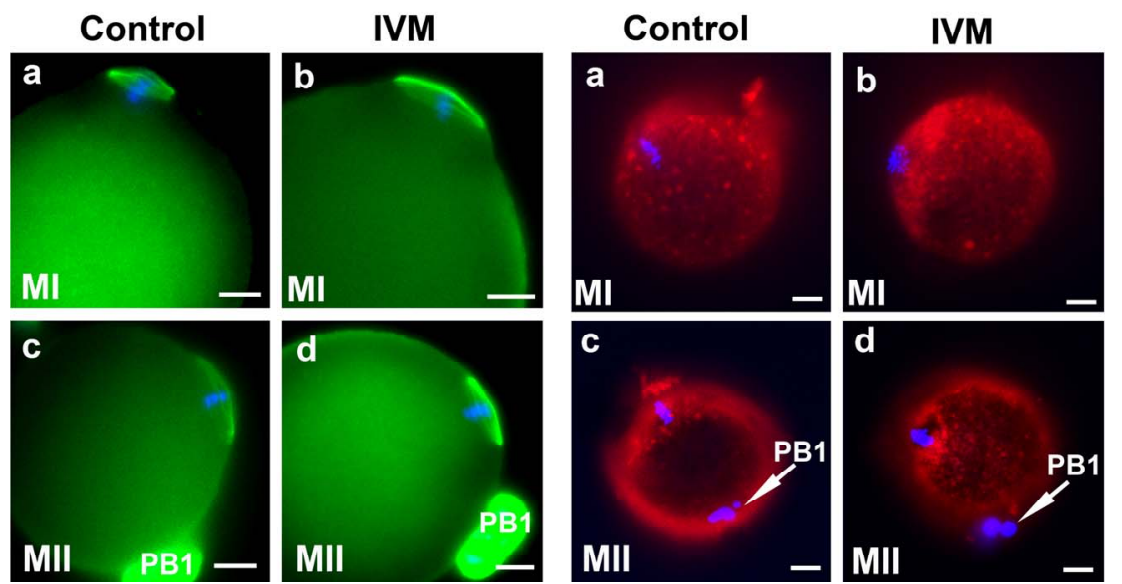

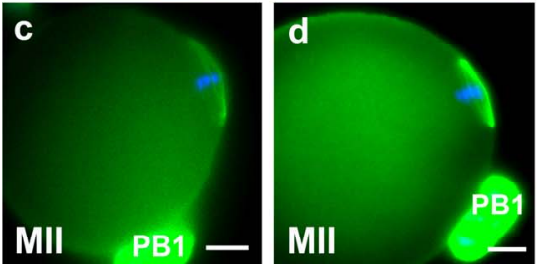

(A)

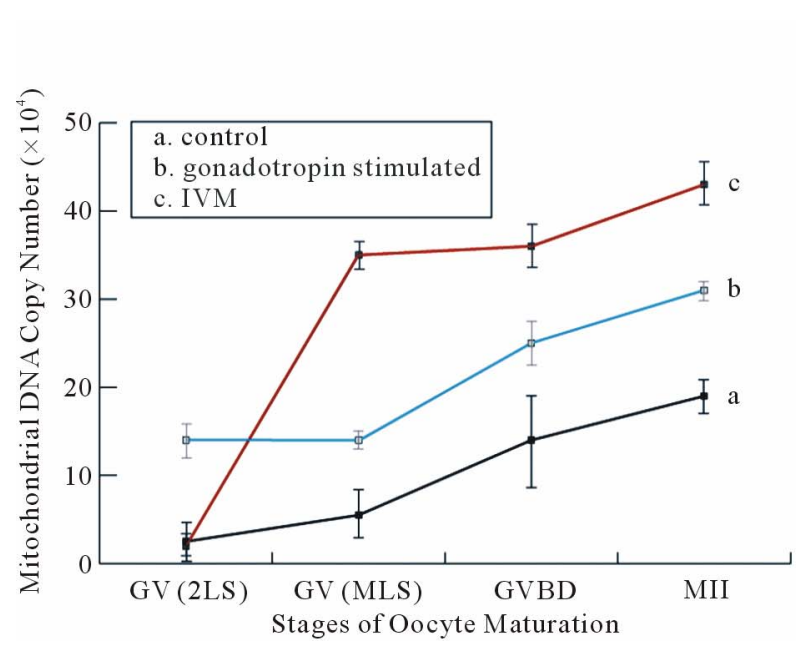

(C)

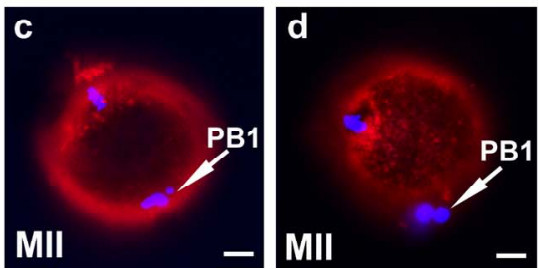

(B)

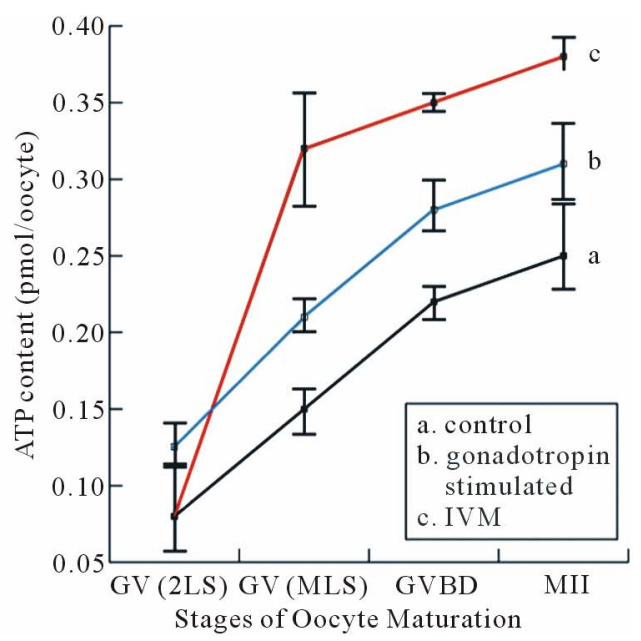

(D)

Figure 5. Cytoplasmic maturation of IVM oocytes. Stage-matched control oocytes from mouse ovaries were included in all assays performed in this study. (A) (a)-(d) Representative immunofluorescence showing the integrity of spindles of MI and MII oocytes by IVM. Nuclei were stained with DAPI; (B) Mitochondrial distribution in the control and IVM oocytes examined by MitoTracker Red staining; (C) Mitochondrial proliferation during the maturation of oocytes from untreated (a), gonadotropin-stimulated (b) and IVM (c) oocytes was measured by quantitative-PCR. Stage-matched oocytes from untreated (nature estrous cycle) and PMSG-stimulated mice were included as controls; (D) ATP content of IVM oocytes of various developmental stages was measured using ENLITEN ATP Assay system. Scale bar, $10 \mu \mathrm{m}$.

similar patterns of changes was observed between the ATP content and the mitochondrial DNA copy number as a function of growth stage (Figures 5(C) and (D)). Oocytes matured by IVM contained a significantly higher level of ATP than the in vivo oocytes $(0.38 \pm 0.02$ $\mathrm{pmol} /$ in vitro oocyte vs $0.25 \pm 0.04 \mathrm{pmole} /$ in vivo oocyte; $\mathrm{p}<0.05)$.

\subsection{In Vitro Fertilization of Matured MII Oocytes}

The capacity of FVB/N-Tg MII oocytes matured in vitro to undergo fertilization, cleavage, blastocyst formation and early embryo development after insemination was determined. The zona-drilled MII oocytes $(\mathrm{n}=32$; Figure 6(A)-a) were inseminated with $\mathrm{C} 57 \mathrm{BL} / 6 \mathrm{~J}$ spermatozoa. Sperm penetration can be seen in all of the MII oocytes under IVF (Figure 6(A)-b). 94\% of spermpenetrated oocytes proceeded to the 2-cell, 3-cell and 4-cell stages (Figures 6(A)-c-e). On day 3 of culture in M16, twenty-five fertilized oocytes developed to the 16-cell, morula or blastocyst stage (Figure 6(A)-f). Immunofluorescence staining of the resulting hybrid blastocysts clearly demonstrated the correct spatial segregation of two committed cell lineages: Nanog-positive inner cell mass and Cdx2-positive outer trophectoderm (Figure 6(B)). Totally, twenty-two viable embryos, regardless of their developmental stages, were transferred 
to the oviducts of two CD-1 pseudo-pregnant recipient mothers. Surprisingly, 17 embryos in total were collected at E9.5 and E11.5 and subjected to lacZ-staining. Figure 6(C) shows eight healthy embryos collected from one pseudo-mother; five of which carried the Umodl1-BAClacZ transgene as confirmed by its expression in the de- veloping inner ears and vertebrae. When the strain-specific D1Mit3 polymorphic marker was examined by genomic PCR, both FVB/N and C57BL6/J alleles were present in the IVF embryos (Figure 6(C)-b). Generation of healthy embryos from IVM oocytes by IVF with a $77 \%$ success rate $(17 / 22)$ strongly demonstrates that our

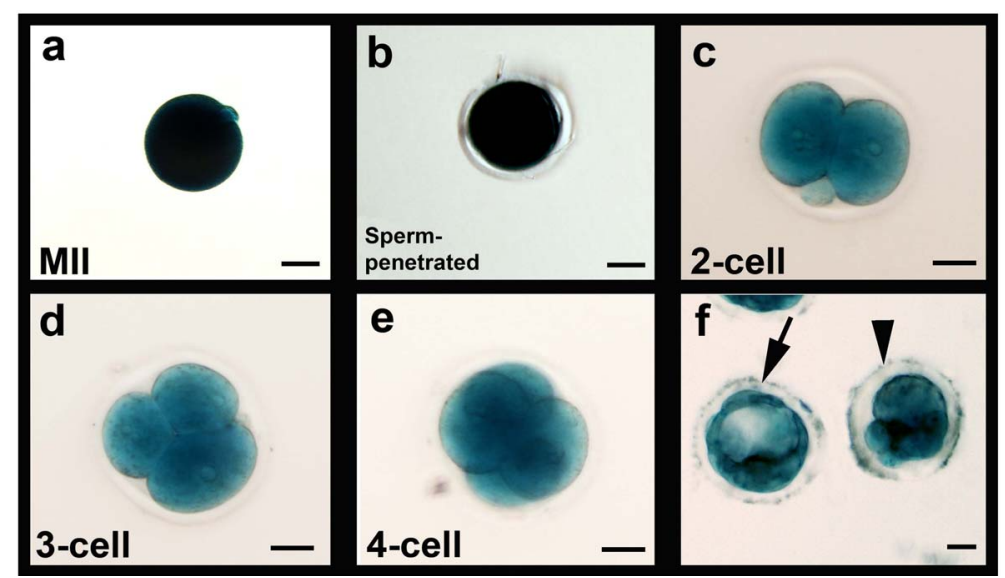

(A)
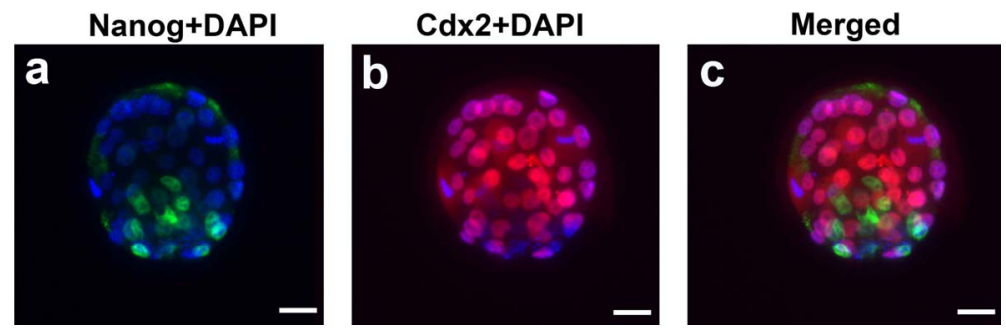

(B)

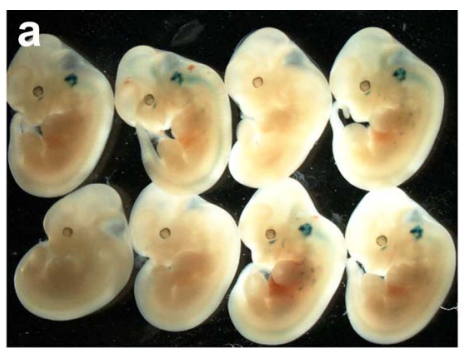

b. Strain-specific D1Mit3 marker

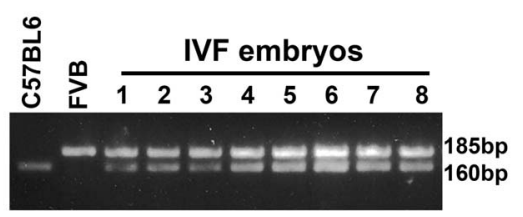

(C)

Figure 6. Developmental potential of IVM oocytes. (A) Matured MII oocytes (a) Can be fertilized by IVF using C57BL/6J sperm; (b) Most of the fertilized oocytes were capable to proceed from 2-cell to blastocyst stage; (c)-(f) Oocytes or embryos were removed at different developmental stages and subjected to lacZ-staining; (B) Correct temporal and spatial expression of Nanog and $\mathrm{Cdx} 2$ maker genes in the inner cell mass and trophectodermal cells of blastocysts derived from fertilized IVM oocytes. Nuclei were visualized by DAPI staining; (C) After being fertilized with C57BL6 sperm, IVM oocyte-derived embryos from 2-cell to morula stages were transferred to the oviducts of CD-1 pseudo-pregnant mothers. (a) Correct expression of Umod11-BAC/lacZ transgene in five E11.5 embryos harvested from one recipient mother; (b) Expected allele distribution among the resulting embryos was confirmed by PCR genotyping of the polymorphic marker D1Mit3. The D1Mit3 primers give rise to the $185 \mathrm{bp} \mathrm{FVB/N-specific} \mathrm{and} \mathrm{the} 160 \mathrm{bp}$ C57BL/6J-specific fragments, respectively. Scale bars, $20 \mu \mathrm{m}$. 
IVM system is reliable to produce oocytes of high quality.

\section{DISCUSSION}

In vivo oocyte maturation is a complex process that requires the acquisition of competence to resume and complete meiotic divisions, accumulation of maternal factors for fertilization and early embryogenesis, and epigenetic reprogramming of the oocyte genome. Great efforts have been made by many researchers to establish in vitro culture models that can closely mimic the in vivo ovarian growth environment. Minimal disturbance of the intricate 3-D architecture of the theca/granulosa-oocyte complex is critical for successful in vitro maturation of follicles/ oocytes, in which the nutrient uptake, as well as paracrine/ endocrine interactions between somatic cells and oocytes can be well sustained $[64,65]$. Spontaneous release of oocytes had always been observed around day 6 of culture in our previously adopted culture systems including both the conventional 2-D and improved alginate-based 3-D systems (data not shown). Both sub-optimal hormonal milieu and spatial restraints, even in the 3-D gel matrices, may contribute to the premature oocyte release and follicle degeneration. In this regard, we employed a two-step culture system in which the isolated singly follicles were first grown in groups by inverted micro-drop suspension culture for 6 days. Once the 3-D architectures were well established as manifested by a healthy follicular expansion and the most susceptible period of oocyte premature release had passed, follicular aggregates were further cultured by the conventional culture method in tissue culture dishes, followed by a 2-day hCG/EGF induction of ovulation. Histological analysis demonstrates that all developmental milestones during folliculogenesis, such as granulosa cell proliferation/differentiation, antral formation, GVBD and PB extrusion, can be faithfully recapitulated by our culture system as the in vivo follicles (Figure 2). This study has achieved a satisfactory result with respect to the oocyte MII maturation rate from inbred mice, and a much better outcome of fertilization and early embryogenesis, when compared with the ground breaking work done by other reputable research groups $[8,29]$. As mentioned before, $~ 90 \%$ (47/50) of GV and degenerated oocytes under IVM were found in follicles that were in direct contact with the tissue culture vessels, suggestive of a different culture milieu from that of the follicles grown on top of them. Despite compromised quality of the enclosed oocytes, the somatic cells including granulosa and theca cells showed no sign of degeneration, and the architecture of the basal membrane was well preserved (Figure 2(B)-k). If the attached follicles were excluded from this study, our success rate of MII maturation would have reached up to $\sim 50 \%$. Therefore, in the same follicular aggregates, spatial variation determined the different fate of the enclosed oocyte. One the other hand, we could not absolutely rule out the possibility that these attached follicles may play some beneficial roles in the maintenance of hormonal environment as well as the 3-D structural support for other follicles. To further improve our IVM efficiency, we propose to coculture follicles with other well characterize immortallized mouse granulosa cells(GC) [66] first by inverted micro-drop suspension culture, followed by transferring these GC-follicle aggregates to micro-droplets of medium covered with oil. Most likely, the immortalized granulosa cells will not only provide the 3-D support for the associated follicles, but also fine tune the culture conditions by secreting essential hormones, growth factors and cytokines.

Among other factors that influence mouse oocyte IVM, hormonal requirements and antioxidant machinery play a critical role in stimulating oocyte nuclear and cytoplasmic maturation. In vitro culture was practically performed in higher oxygen concentration than in vivo environments, leading to an increased of reactive oxygen species (ROS), namely superoxide anion, hydrogen peroxide, and hydroxyl radical $[67,68]$. Elevated ROS production can affect membrane lipids and nuclear/mitochondrial DNA content, and accelerate apoptosis $[69,70]$. Therefore, ROS production and the scavenging activity by antioxidants has to be balanced in both in vivo physiological and in vitro culture conditions. ITS (insulintransferrin-selenium), known to promote nutrient uptake by cells and to detoxify oxygen radicals, was added to our IVM system. Insulin has beneficial effects on granulosa cell proliferation and differentiation, and may improve the cleavage rate as well as developmental potential of early embryos [71]; while selenium and transferring participate in the oocyte antioxidant defense system. Inclusion of FSH is required for the overall well-being of maturing follicles, including the granulose cell proliferation/differentiation as well as the resultant cytoplasmic/ nuclear maturation of oocytes. However, combination of FSH plus high levels of insulin resulted in the precocious differentiation of cumulus cells, and thus was detrimental to oocyte competence [72]. To minimize the deleterious effect, ITS concentration was reduced in our IVM system. Meanwhile, the scavenging ability of the antioxidants was compensated for by increasing the ascorbic acid concentration by $0.25 \mathrm{mM}$ (Note, $\alpha$-MEM contains 0.25 $\mathrm{mM}$ ascorbic acid).

The meiotic spindles consist of microtubules that are formed by polymerization of $\alpha$ - and $\beta$-tubulin $[73,74]$. Formation and subsequent migration of spindles are required for the polarization of mouse oocytes during meiotic maturation, a process essential for asymmetric cell divisions that maximize retention of maternal factors for 
early embryogenesis. Structural disturbance of the meiotic spindles could result in chromosomal dispersion, failure of fertilization and termination of embryogenesis [75]. Integrity of spindles is very sensitive to the culture conditions. Extreme temperature during cryopreservation, or oxidant injury resulted from a sub-optimal medium can damage spindle structures, thereby affecting outcomes of ART $[76,77]$. Despite correct cellular localization and morphology, our IVM spindles showed increased dimensions when compared with the in vivo spindles (Figure 5(A)), which is consistent with the previous report [78]. It further suggests that the follicular milieu was not being faithfully recapitulated under any in vitro conditions reported and further improvement is needed.

Cytoplasmic maturation involves organelle reorganization, increases in the content of $\mathrm{Ca}^{2+}$ stores and antioxidants, and the storage of mRNAs and proteins for the completion of nuclear maturation of oocytes [79,80]. A drastic growth of oocytes during follicular maturation requires well coordinated changes in morphology, metabolic status and gene expression of the developing oocytes/follicles [81]. The energy status, i.e., ATP content, of oocytes is believed to be associated with the developmental competence of embryo [82]. Mitochondria are maternally inherited organelles that supply ATP to the cell by oxidative phosphorylation. Dysfunctional mitochondria and resultant low ATP production are major factors affecting oocyte quality $[83,84]$. Therefore, the dynamics of mitochondrial biogenesis, organization as well as ATP production, which is primarily influenced by hormones, cytokines and energy substrates, has been proposed as one of the critical indicators for the quality of IVM medium. During oocyte maturation, mitochondria are redistributed to distinct regions in response to local energy demands [85]. In GV stage oocytes, mitochondria are clustered surrounding the nucleus, and migrate to the periphery of the oocyte after GVBD (Figure 5(B)-a). Mitochondria of in vivo MII oocytes are predominately located around the meiotic spindle (Figure 5(B)-c). Similar redistribution patterns were observed in our IVM oocytes except that a relatively higher level of mitochondrial signals was retained around the condensed chromosomes of MI oocytes (Figure 5(B)-b) compared with the control oocytes (Figure 5(B)-a), suggesting a mild delay in mitochondrial migration from the nucleus to the peripheral region after GVBD. Unlike spindles whose positioning and cytokinesis are controlled by microfilaments, mitochondrial relocation during oocyte maturation is regulated by microtubules [60]. In general, the correct dynamics of the mitochondria redistribution indicated that the cytoplasmic micro-environment of our IVM oocytes is functioning and competent to support oocyte maturation.
In addition, stronger MitoTracker signals in the IVM oocytes suggest that our IVM system significantly boosted mitochondrial biogenesis and mtDNA replication. Even though as few as $0.01 \%$ of the mitochondria in the oocyte actually contribute the offspring of the next generation, accumulation of "excess" amount of mitochondria is essential for oocyte competence and developmental potential of early embryos [86-91]. Blastomeres that inherit an insufficient amount of mitochondria remain arrested and undergo fragmentation [92]. The critical thresholds of mtDNA copy number for successful fertilization and development of early embryos have been estimated in mature oocytes from a variety of mammals by several groups. In mice, the minimal mtDNA copy number in the oocyte that is essential for post-implantation development is on the order of $40,000-50,000$ copies [89]. In this study, mtDNA copy number of single oocyte at different maturation stage was measured by quantitativePCR. Unlike the in vivo oocytes that showed a progressive increase in its mtDNA copies as the maturation proceeded, the IVM oocytes exhibited a burst of mitochondrial biosynthesis ( $>10$ fold) during the maturation from 2-layered-GV to the multi-layered GV stage, and then reached a steady state of mitochondrial replication in which only a $17.8 \%$ increase was achieved during the transition from GV (MLS) to MII (Figure 5(C)). Consistent with the elevated mitochondrial biosynthesis, the energy status of the IVM oocytes, as manifested by the total ATP content, showed an identical dynamic pattern, and remained at a higher energy level at all stages examined as compared with the controls (Figure 5(D)). From these data, we conclude that our IVM system is prone to mitochondrial biogenesis and mtDNA replication. High mtDNA content in the mature IVM oocytes ensures not only the energy demand by maturing oocytes, but also the faithful transmission of these organelles to all cells of the early embryo. Despite the determination of the threshold value of mitochondria, the optimal range of mitochondrial copies required for oocyte maturation and subsequent embryo development has not been meticulously examined. Emerging evidence has suggested that high mitochondrial DNA copy number has detrimental effects as well on mouse somatic cells [93], which may also be applicable to female gametes. Therefore, in any culture system, mitochondrial biogenesis, mtDNA replication and ATP production must be kept in check to avoid any deleterious impact on the oocyte competence.

Expression patterns of key regulatory genes present us informative clues as to the status of the culture system. Interestingly, all of the oocyte-specific genes examined in this study displayed identical dynamic patterns of expression between the in vivo and in vitro oocytes. Nevertheless, evident are the steady-state levels of genes related to endocrine-signaling and cell-cell interactions 
between the two experimental groups. Decreased levels of FSHR, Glut 1 and Cyp19a1 in the IVM granulosa/ theca cells suggest that a feed-back inhibitory regulation has taken into effect for the self protective purpose by the follicles, in response to the IVM medium that was somehow over rich in nutrients, hormones and cytokines. Difference in expression can also be seen in some genes encoding ECM and cell-cell junction proteins between the IVM and control follicles/oocytes (Figure 4), further supporting that, no matter how good it was manipulated, the IVM system can not fully reiterate all physiological events as in vivo. Once these limiting components were identified, they can be used as breakthrough points to improve the IVM efficiency.

In addition to its critical roles in morphogenesis during early embryogenesis and male germ cell development, retinoic acid (RA) is actively involved in female reproduction. At the early embryonic stage of 13.5, RA produced by the mesonephros is required for entry into meiosis of female gonocytes, the committed primordial germ cells (PGCs). Whether RA is able to promote the MI to MII transition in oocytes is elusive. But, the stimulatory effect of RA in oocyte maturation has been reported in many animal model systems [46,47,94,95]. RA exerts its functions on oocyte competence not only by its antioxidant properties, but also by its regulatory effects on the down-stream genes including gonadotropin receptors, cyclooxygenase- 2 and nitric oxide synthase in cumulus-granulosa cells. Based on the previous studies, we included RA at $2 \mu \mathrm{m}$ in our culture medium, which may partly contribute the enhanced rates in fertilization and embryogenesis in our IVM system. Despite its positive role in mouse oocyte maturation, precautions have to be taken before its application to humans because of its carcinogenicity. Even with mouse oocytes, prolonged culture in medium supplemented with RA could induce more parthenogenetic embryos.

\section{ACKNOWLEDGEMENTS}

We would like to thank the Ronald O. Perelman and Claudia Cohen Center for Reproductive Medicine at Weill Medical College of Cornell University for funding this project.

\section{REFERENCES}

[1] Horwath, D., et al. (2007) Subsequent therapeutic options and outcome in couples who fail to fertilize despite in vitro fertilization (IVF) and intracytoplasmic sperm injection (ICSI). Clinical \& Experimental Obstetrics \& Gynecology, 34, 109-110.

[2] Singh, L.K., Davies, M. and Chatterjee, R. (2005) Fertility in female cancer survivors: Pathophysiology, preservation and the role of ovarian reserve testing. Human Reproduction Update, 11, 69-89. doi:10.1093/humupd/dmh052

[3] Suikkari, A.M. and Soderstrom-Anttila, V. (2007) In-vitro maturation of eggs: Is it really useful? Best Practice \& Research Clinical Obstetrics \& Gynaecology, 21, 145155. doi:10.1016/j.bpobgyn.2006.09.003

[4] Cha, K.Y., et al. (2005) Obstetric outcome of patients with polycystic ovary syndrome treated by in vitro maturation and in vitro fertilization-embryo transfer. Fertility and Sterility, 83, 1461-1465. doi:10.1016/j.fertnstert.2004.11.044

[5] Gilchrist, R.B. and Thompson, J.G. (2007) Oocyte maturation: Emerging concepts and technologies to improve developmental potential in vitro. Theriogenology, 67, 6-15. doi:10.1016/j.theriogenology.2006.09.027

[6] Hashimoto, S. (2009) Application of in vitro maturation to assisted reproductive technology. Journal of Reproduction and Development, 55, 1-10. doi: $10.1262 /$ jrd.20127

[7] Eppig, J.J. and Schroeder, A.C. (1989) Capacity of mouse oocytes from preantral follicles to undergo embryogenesis and development to live young after growth, maturation, and fertilization in vitro. Biology of Reproduction, 41, 268-276. doi:10.1095/biolreprod41.2.268

[8] Cortvrindt, R., Smitz, J. and Van Steirteghem, A.C. (1996) In-vitro maturation, fertilization and embryo development of immature oocytes from early preantral follicles from prepuberal mice in a simplified culture system. Human Reproduction, 11, 2656-2666.

[9] Xu, M., et al. (2006) Tissue-engineered follicles produce live, fertile offspring. Tissue Engineering, 12, 2739-2746. doi:10.1089/ten.2006.12.2739

[10] Desai, N., et al. (2010) Three-dimensional in vitro follicle growth: Overview of culture models, biomaterials, design parameters and future directions. Reproductive Biology and Endocrinology, 8, 119. doi:10.1186/1477-7827-8-119

[11] Nayudu, P.L. and Osborn, S.M. (1992) Factors influencing the rate of preantral and antral growth of mouse ovarian follicles in vitro. Journal of Reproduction and Fertility, 95, 349-362. doi:10.1530/irf.0.0950349

[12] Boland, N.I., et al. (1993) Pattern of lactate production and steroidogenesis during growth and maturation of mouse ovarian follicles in vitro. Biology of Reproduction, 48, 798-806. doi:10.1095/biolreprod48.4.798

[13] Xu, M., et al. (2009) Encapsulated three-dimensional culture supports development of nonhuman primate secondary follicles. Biology of Reproduction, 81, 587-594. doi:10.1095/biolreprod.108.074732

[14] Eppig, J.J. (1991) Intercommunication between mammalian oocytes and companion somatic cells. Bioessays, 13, 569574. doi:10.1002/bies.950131105

[15] Carabatsos, M.J., et al. (2000) Oocyte-granulosa cell heterologous gap junctions are required for the coordination of nuclear and cytoplasmic meiotic competence. Developmental Biology, 226, 167-179. doi:10.1006/dbio.2000.9863

[16] Albertini, D.F., et al. (2001) Cellular basis for paracrine regulation of ovarian follicle development. Reproduction, 
121, 647-653. doi:10.1530/rep.0.1210647

[17] McGee, E.A. and Hsueh, A.J. (2000) Initial and cyclic recruitment of ovarian follicles. Endocrine Reviews, 21, 200-214. doi:10.1210/er.21.2.200

[18] McNatty, K.P., et al. (2007) Control of ovarian follicular development to the gonadotrophin-dependent phase: A 2006 perspective. Society for Reproduction and Fertility Suppleent, 64, 55-68.

[19] Craig, J., et al. (2007) Gonadotropin and intra-ovarian signals regulating follicle development and atresia: The delicate balance between life and death. Front Biosci, 12, 3628-3639. doi: 10.2741/2339

[20] Kumar, T.R., et al. (1997) Follicle stimulating hormone is required for ovarian follicle maturation but not male fertility. Nature Genetics, 15, 201-204. doi:10.1038/ng0297-201

[21] Hirshfield, A.N. (1991) Development of follicles in the mammalian ovary. International Review of Cytology, 124, 43-101. doi:10.1016/S0074-7696(08)61524-7

[22] Gilchrist, R.B., Ritter, L.J. and Armstrong, D.T. (2004) Oocyte-somatic cell interactions during follicle development in mammals. Animal Reproduction Science, 82-83, 431-446. doi:10.1016/j.anireprosci.2004.05.017

[23] Oktem, O. and Urman, B. (2010) Understanding follicle growth in vivo. Human Reproduction, 25, 2944-2954. doi:10.1093/humrep/deq275

[24] Eppig, J.J., et al. (1998) Factors affecting the developmental competence of mouse oocytes grown in vitro: Follicle-stimulating hormone and insulin. Biology of Reproduction, 59, 1445-1453. doi:10.1095/biolreprod59.6.1445

[25] Sanfins, A., et al. (2003) Distinctions in meiotic spindle structure and assembly during in vitro and in vivo maturation of mouse oocytes. Biology of Reproduction, 69, 2059-2067. doi:10.1095/biolreprod.103.020537

[26] Sanfins, A., et al. (2004) Meiotic spindle morphogenesis in in vivo and in vitro matured mouse oocytes: Insights into the relationship between nuclear and cytoplasmic quality. Human Reproduction, 19, 2889-2899. doi:10.1093/humrep/deh528

[27] Erickson, G.F. and Sorensen, R.A. (1974) In vitro maturation of mouse oocytes isolated from late, middle, and pre-antral graafian follicles. Journal of Experimental Zoology, 190, 123-127. doi:10.1002/jez.1401900112

[28] Sorensen, R.A. and Wassarman, P.M. (1976) Relationship between growth and meiotic maturation of the mouse oocyte. Developmental Biology, 50, 531-536. doi:10.1016/0012-1606(76)90172-X

[29] O'Brien, M.J., Pendola, J.K. and Eppig, J.J. (2003) A revised protocol for in vitro development of mouse oocytes from primordial follicles dramatically improves their developmental competence. Biology of Reproduction, 68, 1682-1686. doi:10.1095/biolreprod.102.013029

[30] Adam, A.A., et al. (2004) In vitro culture of mouse preantral follicles using membrane inserts and developmental competence of in vitro ovulated oocytes. Journal of Reproduction and Development, 50, 579-586. doi:10.1262/jrd.50.579
[31] De Sousa, P.A., et al. (1998) Temporal patterns of embryonic gene expression and their dependence on oogenetic factors. Theriogenology, 49, 115-128. doi:10.1016/S0093-691X(97)00406-8

[32] De Vant'ery, C., et al. (1996) An accumulation of p $34 \mathrm{cdc} 2$ at the end of mouse oocyte growth correlates with the acquisition of meiotic competence. Developmental Biology, 174, 335-344. doi:10.1006/dbio.1996.0078

[33] Holt, J.E., et al. (2011) The APC/C activator FZR1 coordinates the timing of meiotic resumption during prophase I arrest in mammalian oocytes. Development, 138, $905-$ 913. doi:10.1242/dev.059022

[34] Eppig, J.J. and Wigglesworth, K. (1994) Atypical maturation of oocytes of strain I/LnJ mice. Human Reproduction, 9, 1136-1142.

[35] Silver, L. (1995) Reproduction and breeding. Mouse genetics, concepts and applications. Oxford University Press, New York, 62-63.

[36] Kaleta, E. (1977) Influence of genetic factors on the fertilization of mouse ova in vitro. Journal of Reproduction \& Fertility, 51, 375-381. doi:10.1530/jrf.0.0510375

[37] Luckett, D.C. and Mukherjee, A.B. (1986) Embryonic characteristics in superovulated mouse strains. Comparative analyses of the incidence of chromosomal aberrations, morphological malformations, and mortality of embryos from two strains of superovulated mice. Journal of $\mathrm{He}$ redity, 77, 39-42.

[38] Gao, S., et al. (2004) Genetic variation in oocyte phenotype revealed through parthenogenesis and cloning: Correlation with differences in pronuclear epigenetic modification. Biology of Reproduction, 70, 1162-1170. doi:10.1095/biolreprod.103.024216

[39] Ibanez, E., Albertini, D.F. and Overstrom, E.W. (2005) Effect of genetic background and activating stimulus on the timing of meiotic cell cycle progression in parthenogenetically activated mouse oocytes. Reproduction, 129, 27-38. doi:10.1530/rep.1.00452

[40] Suzuki, O., et al. (1996) Development in vitro of preimplantation embryos from 55 mouse strains. Reproduction, Fertility and Development, 8, 975-980. doi:10.1530/rep.1.00452

[41] Spearow, J.L., et al. (1999) Genetic variation in susceptibility to endocrine disruption by estrogen in mice. Science, 285, 1259-1261. doi:10.1126/science.285.5431.1259

[42] Polanski, Z. (1997) Genetic background of the differences in timing of meiotic maturation in mouse oocytes: A study using recombinant inbred strains. Journal of Reproduction \& Fertility, 109, 109-114. doi:10.1530/jrf.0.1090109

[43] Wang, W. and Lufkin, T. (2000) The murine Otp homeobox gene plays an essential role in the specification of neuronal cell lineages in the developing hypothalamus. Developmental Biology, 227, 432-449. doi:10.1006/dbio.2000.9902

[44] Wang, W., et al. (2001) Hmx2 homeobox gene control of murine vestibular morphogenesis. Development, 128, 5017- 
5029.

[45] Kelley, K.A., (2010) Enhancement of IVF in the mouse by zona-drilling. Methods in Enzymology, 476, 229-250. doi:10.1016/S0076-6879(10)76013-4

[46] Alminana, C., et al. (2008) In vitro maturation of porcine oocytes with retinoids improves embryonic development. Reproduction, Fertility and Development, 20, 483-489. doi:10.1071/RD07175

[47] Ikeda, S., et al. (2005) The roles of vitamin A for cytoplasmic maturation of bovine oocytes. Journal of Reproduction and Development, 51, 23-35. doi:10.1262/jrd.51.23

[48] Hattori, M.A., Kato, Y. and Fujihara, N. (2002) Retinoic acid suppression of endothelial nitric oxide synthase in porcine oocyte. Canadian Journal of Physiology and Pharmacology, 80, 777-782. doi:10.1139/y02-099

[49] Tahaei, L.S., et al. (2011) Effects of retinoic acid on maturation of immature mouse oocytes in the presence and absence of a granulosa cell co-culture system. Journal of Assisted Reproduction and Genetics, 28, 553-558. doi:10.1007/s10815-011-9579-8

[50] Gilchrist, R.B., et al. (2006) Molecular basis of oocyte-paracrine signalling that promotes granulosa cell proliferation. Journal of Cell Science, 119, 3811-3821. doi: $10.1242 /$ jes. 03105

[51] Orisaka, M., et al. (2009) Growth differentiation factor 9 promotes rat preantral follicle growth by up-regulating follicular androgen biosynthesis. Endocrinology, 150, 2740-2748. doi:10.1210/en.2008-1536

[52] Thomas, F.H., et al. (2001) Effect of ascorbic acid on health and morphology of bovine preantral follicles during long-term culture. Reproduction, 122, 487-495. doi:10.1530/rep.0.1220487

[53] Fulop, C., et al. (2003) Impaired cumulus mucification and female sterility in tumor necrosis factor-induced protein-6 deficient mice. Development, 130, 2253-2261. doi:10.1242/dev.00422

[54] Ny, T., Wahlberg, P. and Brandstrom, I.J. (2002) Matrix remodeling in the ovary: Regulation and functional role of the plasminogen activator and matrix metalloproteinase systems. Molecular and Cellular Endocrinology, 187, 29-38. doi:10.1016/S0303-7207(01)00711-0

[55] Kerr, J.F., Wyllie, A.H. and Currie, A.R. (1972) Apoptosis: A basic biological phenomenon with wide-ranging implications in tissue kinetics. British Journal of Cancer, 26, 239-257. doi:10.1038/bjc.1972.33

[56] Roberts, R., et al. (2005) Follicle-stimulating hormone affects metaphase I chromosome alignment and increases aneuploidy in mouse oocytes matured in vitro. Biology of Reproduction, 72, 107-118. doi:10.1095/biolreprod.104.032003

[57] Combelles, C.M., et al. (2002) Assessment of nuclear and cytoplasmic maturation in in-vitro matured human oocytes. Human Reproduction, 17, 1006-1016. doi:10.1093/humrep/17.4.1006

[58] Vanhoutte, L., et al. (2007) Nuclear and cytoplasmic maturation of in vitro matured human oocytes after temporary nuclear arrest by phosphodiesterase 3-inhibitor.
Human Reproduction, 22, 1239-1246. doi:10.1093/humrep/dem007

[59] Mullen, S.F., Rosenbaum, M. and Critser, J.K. (2007) The effect of osmotic stress on the cell volume, metaphase II spindle and developmental potential of in vitro matured porcine oocytes. Cryobiology, 54, 281-289. doi:10.1016/j.cryobiol.2007.03.005

[60] Sun, Q.Y., et al. (2001) Translocation of active mitochondria during pig oocyte maturation, fertilization and early embryo development in vitro. Reproduction, 122, 155-163. doi:10.1530/rep.0.1220155

[61] Adona, P.R., et al. (2008) Prematuration of bovine oocytes with butyrolactone I: Effects on meiosis progression, cytoskeleton, organelle distribution and embryo development. Animal Reproduction Science, 108, 49-65. doi:10.1016/j.anireprosci.2007.07.002

[62] Wassarman, P.M., et al. (1979) Meiotic maturation of mouse oocytes in vitro. Advances in Experimental Medicine and Biology, 112, 251-268.

[63] Van Blerkom, J. (1991) Microtubule mediation of cytoplasmic and nuclear maturation during the early stages of resumed meiosis in cultured mouse oocytes. Proceedings of the National Academy of Sciences of the United States of America, 88, 5031-5035. doi:10.1073/pnas.88.11.5031

[64] Buccione, R., Schroeder, A.C. and Eppig, J.J. (1990) Interactions between somatic cells and germ cells throughout mammalian oogenesis. Biology of Reproduction, 43, 543-547. doi:10.1095/biolreprod43.4.543

[65] Su, Y.Q., Sugiura, K. and Eppig, J.J. (2009) Mouse oocyte control of granulosa cell development and function: Paracrine regulation of cumulus cell metabolism. Seminars in Reproductive Medicine, 27, 32-42. doi:10.1055/s-0028-1108008

[66] Vanderstichele, H., et al. (1994) Secretion of steroids, growth factors, and cytokines by immortalized mouse granulosa cell lines. Biology of Reproduction, 50, 11901202. doi:10.1095/biolreprod50.5.1190

[67] Halliwell, B. and Cross, C.E. (1994) Oxygen-derived species: Their relation to human disease and environmental stress. Environmental Health Perspectives, 102, 5-12.

[68] Nasr-Esfahani, M.H., Aitken, J.R. and Johnson, M.H. (1990) Hydrogen peroxide levels in mouse oocytes and early cleavage stage embryos developed in vitro or in vivo. Development, 109, 501-507.

[69] Lopes, S., et al. (1998) Reactive oxygen species: Potential cause for DNA fragmentation in human spermatozoa. Human Reproduction, 13, 896-900. doi:10.1093/humrep/13.4.896

[70] Kowaltowski, A.J. and Vercesi, A.E. (1999) Mitochondrial damage induced by conditions of oxidative stress. Free Radical Biology and Medicine, 26, 463-471. doi:10.1016/S0891-5849(98)00216-0

[71] Lee, M.S., et al. (2005) The beneficial effects of insulin and metformin on in vitro developmental potential of porcine oocytes and embryos. Biology of Reproduction, 73, 1264-1268. doi:10.1095/biolreprod.105.041186 
[72] Eppig, J.J., et al. (2000) Conditions that affect acquisition of developmental competence by mouse oocytes in vitro: FSH, insulin, glucose and ascorbic acid. Molecular and Cellular Endocrinology, 163, 109-116. doi:10.1016/S0303-7207(99)00247-6

[73] Pickering, S.J., et al. (1988) Cytoskeletal organization in fresh, aged and spontaneously activated human oocytes. Human Reproduction, 3, 978-989.

[74] Tao, W., et al. (2005) Induction of apoptosis by an inhibitor of the mitotic kinesin KSP requires both activation of the spindle assembly checkpoint and mitotic slippage. Cancer Cell, 8, 49-59. doi:10.1016/j.ccr.2005.06.003

[75] Eroglu, A., et al. (1998) Cytoskeleton and polyploidy after maturation and fertilization of cryopreserved germinal vesicle-stage mouse oocytes. Journal of Assisted Reproduction and Genetics, 15, 447-454. doi:10.1007/BF02744940

[76] Rienzi, L., et al. (2003) Relationship between meiotic spindle location with regard to the polar body position and oocyte developmental potential after ICSI. Human Reproduction, 18, 1289-1293. doi:10.1093/humrep/deg274

[77] Rienzi, L., et al. (2005) Meiotic spindle visualization in living human oocytes. Reproductive BioMedicine Online, 10, 192-198. doi:10.1016/S1472-6483(10)60940-6

[78] Ibanez, E., et al. (2005) Genetic strain variations in the metaphase-II phenotype of mouse oocytes matured in vivo or in vitro. Reproduction, 130, 845-855. doi:10.1530/rep.1.00558

[79] Guerin, P., El Mouatassim, S. and Menezo, Y. (2001) Oxidative stress and protection against reactive oxygen species in the pre-implantation embryo and its surroundings. Human Reproduction Update, 7, 175-189. doi:10.1093/humupd/7.2.175

[80] Ferreira, E.M., et al. (2009) Cytoplasmic maturation of bovine oocytes: Structural and biochemical modifications and acquisition of developmental competence. Theriogenology, 71, 836-848. doi:10.1016/j.theriogenology.2008.10.023

[81] Gosden, R., Krapez, J. and Briggs, D. (1997) Growth and development of the mammalian oocyte. Bioessays, 19, 875-882. doi:10.1002/bies.950191007

[82] Van Blerkom, J., Sinclair, J. and Davis, P. (1998) Mitochondrial transfer between oocytes: potential applications of mitochondrial donation and the issue of heteroplasmy. Human Reproduction, 13, 2857-2868.

[83] Zeng, H.T., et al. (2007) Low mitochondrial DNA and ATP contents contribute to the absence of birefringent spindle imaged with PolScope in in vitro matured human oocytes. Human Reproduction, 22, 1681-1686.

\section{doi:10.1093/humrep/dem070}

[84] Takeuchi, T., et al. (2005) Effect of treating induced mitochondrial damage on embryonic development and epigenesis. Biology of Reproduction, 72, 584-592. doi:10.1095/biolreprod.104.032391

[85] Bavister, B.D. and Squirrell, J.M. (2000) Mitochondrial distribution and function in oocytes and early embryos. Human Reproduction, 15, 189-198.

[86] El Shourbagy, S.H., et al. (2006) Mitochondria directly influence fertilisation outcome in the pig. Reproduction, 131, 233-245. doi:10.1530/rep.1.00551

[87] Santos, T.A., El Shourbagy, S. and St. John, J.C. (2006) Mitochondrial content reflects oocyte variability and fertilization outcome. Fertility and Sterility, 85, 584-591. doi:10.1016/j.fertnstert.2005.09.017

[88] Dumollard, R., et al. (2008) Regulation of cytosolic and mitochondrial ATP levels in mouse eggs and zygotes. Developmental Biology, 316, 431-440. doi:10.1016/j.ydbio.2008.02.004

[89] Wai, T., et al. (2010) The role of mitochondrial DNA copy number in mammalian fertility. Biology of Reproduction, 83, 52-62. doi:10.1095/biolreprod.109.080887

[90] Reynier, P., et al. (2001) Mitochondrial DNA content affects the fertilizability of human oocytes. Molecular Human Reproduction, 7, 425-429. doi:10.1093/molehr $/ 7.5 .425$

[91] Wilding, M., et al. (2001) Mitochondrial aggregation patterns and activity in human oocytes and preimplantation embryos. Human Reproduction, 16, 909-917. doi:10.1093/humrep/16.5.909

[92] Van Blerkom, J., Davis, P. and Alexander, S. (2000) Differential mitochondrial distribution in human pronuclear embryos leads to disproportionate inheritance between blastomeres: Relationship to microtubular organization, ATP content and competence. Human Reproduction, 15, 2621-2633. doi:10.1093/humrep/15.12.2621

[93] Ylikallio, E., et al. (2010) High mitochondrial DNA copy number has detrimental effects in mice. Human Molecular Genetics, 19, 2695-2705. doi: $10.1093 / \mathrm{hmg} / \mathrm{ddq} 163$

[94] Duque, P., et al. (2002) Enhancement of developmental capacity of meiotically inhibited bovine oocytes by retinoic acid. Human Reproduction, 17, 2706-2714. doi:10.1093/humrep/17.10.2706

[95] Atikuzzaman, M., et al. (2011) The 9-cis retinoic acid signaling pathway and its regulation of prostaglandinendoperoxide synthase 2 during in vitro maturation of pig cumulus cell-oocyte complexes and effects on parthenogenetic embryo production. Biology of Reproduction, 84, 1272-1281. doi:10.1095/biolreprod.110.086595 